\title{
Paneth Cell Ablation Aggravates Pancreatic and Intestinal Injuries in a Rat Model of Acute Necrotizing Pancreatitis after Normal and High-Fat Diet
}

\author{
Yuecheng Guo, ${ }^{1,2}$ Chunlan Huang, ${ }^{1}$ Liyan Liu, ${ }^{3}$ Xinyuan Fu, ${ }^{4}$ Yingying $\mathrm{Lu},{ }^{1}$ \\ Junyuan Zheng, ${ }^{1,2}$ Qixiang Mei, ${ }^{1,2}$ Zehua Huang, ${ }^{1,2}$ Junjie Fan, ${ }^{1}$ Lungen Lu ${ }^{1}{ }^{1}$ \\ and Yue Zeng $\mathbb{D}^{1,2}$ \\ ${ }^{1}$ Department of Gastroenterology, Shanghai General Hospital, Shanghai Jiao Tong University School of Medicine, Shanghai, China \\ ${ }^{2}$ Shanghai Key Laboratory of Pancreatic Diseases, Shanghai Jiao Tong University School of Medicine, Shanghai, China \\ ${ }^{3}$ International Medical Care Center, Shanghai General Hospital, Shanghai Jiao Tong University School of Medicine, Shanghai, China \\ ${ }^{4}$ Shanghai Minhang High School, Shanghai, China
}

Correspondence should be addressed to Lungen Lu; lungen.lu@shgh.cn and Yue Zeng; zengyue1592@yahoo.com

Received 27 June 2019; Revised 28 August 2019; Accepted 13 September 2019; Published 11 November 2019

Academic Editor: Joilson O. Martins

Copyright (C) 2019 Yuecheng Guo et al. This is an open access article distributed under the Creative Commons Attribution License, which permits unrestricted use, distribution, and reproduction in any medium, provided the original work is properly cited.

\begin{abstract}
We previously reported that acute necrotizing pancreatitis (ANP) after normal or high-fat diet is associated with a decreased number of Paneth cells in ileal crypts. Here, we ablated Paneth cells in a rat model of ANP after normal and high-fat diet to investigate the effects on disease symptoms. Adult male Sprague-Dawley rats received standard rat chow or a high-fat diet for 2 weeks, after which they were treated with dithizone to deplete Paneth cells. Six hours later, ANP was established by retrograde injection of sodium taurocholate into the biliopancreatic duct. Rats were sacrificed at 6,12 , and $24 \mathrm{~h}$ for assessment. We found dithizone aggravated ANP-associated pathological injuries to the pancreas and ileum in rats on high-fat or standard diets. Lysozyme expression in ileal crypts was decreased, while serum inflammatory cytokines (TNF $\alpha$, IL-1 $\beta$, and IL-17A) and intestinal permeability (serum DAO activity and D-lactate) were increased. Expression of tight junction proteins (claudin-1, zo-1, and occludin) was decreased. Using high-throughput $16 \mathrm{~S}$ rRNA sequencing, we found dithizone reduced microbiota diversity and altered microbiota composition in rats on high-fat or standard diets. Dithizone decreased fecal short-chain fatty acids (SCFAs) in rats on high-fat or standard diets. Changes in intestinal microbiota correlated significantly with SCFAs, lysozyme, DAO activity, D-lactate, inflammatory cytokines, and pathological injury to the pancreas and ileum in rats on high-fat or standard diets. In conclusion, ablation of Paneth cells exacerbates pancreatic and intestinal injuries in ANP after normal and high-fat diet. These symptoms may be related to changes in the intestinal microbiota.
\end{abstract}

\section{Introduction}

Acute necrotizing pancreatitis (ANP) is a severe form of acute pancreatitis characterized by inflammation associated with necrosis in and around the pancreas. High-fat diet increases the risk of ANP [1] and can exacerbate ANP inflammation and increase complication rates [2]. Dysbiosis of intestinal microbiota has also been reported to correlate with a proinflammatory response in ANP patients [3]. We have previously reported that ANP associated with high-fat diet intake is associated with severe systemic inflammation, decreased Paneth cells in ileal crypts, altered microbiota composition, and decreased microbial diversity $[4,5]$. However, the biological significance of these symptoms and their contribution to ANP are unclear.

Paneth cells are zinc-containing cells at the base of the intestinal crypts that play a vital role in intestinal barrier function [6]. Paneth cells regulate intestinal homeostasis by synthesizing and releasing antimicrobial peptides such as lysozyme and $\alpha$-defensins [7], and thus, disrupting Paneth 
cell secretion can lead to inflammatory disease [8]. Specific deficiency of defensins excreted from Paneth cells during ileal Crohn's disease may compromise innate immune defenses of the ileal mucosa and perpetuate this disease [9]. A high-fat diet increases serum lipid levels [10] and decreases the number of Paneth cells, which in turn reduces the level of antimicrobial peptides in the intestine and alters microbiota structure [11]. Short-chain fatty acids (SCFAs) are produced by microbial fermentation of undigested dietary carbohydrates in the intestine [12] and can protect the intestinal barrier by upregulating epithelial tight junction proteins (TJPs) $[13,14]$.

In this study, we used dithizone, a chelator of zinc complexes [15], to deplete Paneth cells in rats with ANP after normal and high-fat diet. Effects on intestinal barrier function, microbiota structure, SCFAs, and pancreatic and ileal pathological injuries were examined.

\section{Materials and Methods}

2.1. Animals. Male Sprague-Dawley rats (4 weeks old) were obtained from Shanghai SLAC Laboratory Animal Co. (Shanghai, China). Rats were housed in groups of 5 at $24^{\circ} \mathrm{C}$ with a 12/12-hour light-dark cycle and given free access to water and chow. This study was carried out in accordance with the recommendations of the National Animal Protection Guidelines approved by the local animal ethics committee. The protocol was approved by the Animal Care and Use Committee of Shanghai Jiao Tong University.

2.2. Dithizone Treatment and ANP Induction. Rats in the standard diet groups were maintained on standard (STD) diet (Shanghai SLAC Laboratory Animal Co., China), while those in the high-fat- (HF-) fed groups were maintained on high-fat diet (STD diet supplemented with $20 \%$ lard and $3 \%$ cholesterol; Shanghai SLAC Laboratory Animal Co. China) $[5,16]$.

After 2 weeks of feeding, rats in each dietary group were randomly divided into 2 equal groups ( $n=24$ per group). Rats in the dithizone groups (DI+STD and DI+HF) were intravenously injected with $100 \mathrm{mg} / \mathrm{kg}$ dithizone (SigmaAldrich, USA), while nondithizone groups (STD and HF) were injected with an equal volume of saline. Six hours after injection, all rats were anesthetized by intraperitoneal injection of $0.05 \mathrm{mg} / \mathrm{kg}$ sodium pentobarbital (Shanghai Yuyan Instruments, China). Rats in each group were then infused with $3.5 \%$ sodium taurocholate solution (Sigma-Aldrich, USA) at a volume of $0.1 \mathrm{ml} / 100 \mathrm{~g}$ via the biliopancreatic duct at the speed of $0.2 \mathrm{ml} / \mathrm{min}$ to induce ANP [4]. Each group also included control rats that were given a sham biliopancreatic infusion of saline without ANP induction.

Rats were sacrificed by decapitation at 6,12 , and $24 \mathrm{~h}$ after infusion for histological assessment of the pancreas and distal ileum ( $n=8$ per treatment per time point). Blood samples were collected from the abdominal aorta. Segments of the distal ileum and the pancreas were isolated, flashfrozen in liquid nitrogen, and stored at $-80^{\circ} \mathrm{C}$. Freshly excreted feces were also collected from rats for analysis of SCFAs before they were anesthetized.
2.3. Histological Analysis. Pancreatic and distal ileal tissues were fixed in $4 \%$ paraformaldehyde, dehydrated, embedded in paraffin, and cut into $4 \mu \mathrm{m}$ sections. Sections were stained with hematoxylin and eosin (H\&E, Servicebio, China) and examined under a light microscope (DM5500 B, LEICA, Germany) by two pathologists who were blinded to treatment group. Pancreatic injury was scored using criteria reported by Schmidt et al. [17], while injury to the distal ileum was scored as described by Chiu et al. [18]. Paneth cells were counted in at least 10 ileal crypts per section. Three histological sections were analyzed per animal in the histological analyses.

2.4. Serum Biochemistry. Serum triglyceride (TG) and total cholesterol (TC) levels were analyzed using the TG and T-CHO kits (product nos. A110-1 and A111-1, Nanjing Jiancheng Bioengineering Institute, Nanjing, China) according to manufacturer's protocols. D-lactate concentration was determined using the D-LA ELISA kit (Nanjing Jiancheng Bioengineering Institute). DAO activity was measured using the diamine oxidase (DAO) assay kit (Nanjing Jiancheng Bioengineering Institute) according to previously published protocols [5].

2.5. ELISA. Serum TNF $\alpha$, IL- $1 \beta$, and IL-17 levels were measured using the TNF Alpha Rat ELISA kit, IL-1 $\beta$ Rat ELISA kit, and IL-17A Rat ELISA kit (eBioscience, USA) according to manufacturer's protocols. All samples were measured in duplicate.

2.6. Immunohistochemistry. Tissue sections from the distal ileum were deparaffinized, and the antigens were retrieved with EDTA antigen retrieval buffer ( $\mathrm{pH} 9.0)$. After extensive washing in phosphate-buffered saline (PBS, $\mathrm{pH}$ 7.4), the sections were quenched with $3 \%$ hydrogen peroxide, blocked with $3 \%$ bovine serum albumin at room temperature for $30 \mathrm{~min}$, and incubated with 1:100 dilutions of primary antibody against claudin-1 (Abcam, USA), ZO-1 (Proteintech Group, USA), or occludin (Abcam, USA) at $4^{\circ} \mathrm{C}$ overnight. The next day, the sections were washed and incubated with horseradish peroxidase-labeled secondary antibody $(1: 200$, Servicebio, Wuhan, China) for $50 \mathrm{~min}$ at room temperature. The slides were placed in PBS, washed 3 times on a decolorizing shaker for $5 \mathrm{~min}$ per wash, and visualized with diaminobenzidine $(1: 100,50 \mathrm{ul}$ per slide; DAB, DAKO, Denmark). Slides were visualized using a light microscope (DM5500 B, LEICA, Germany), and images were analyzed using Image-Pro Plus 6.0 (Media Cybernetics, USA). Three histological sections were analyzed per animal in the immunohistochemical analyses.

2.7. Western Blot. Tissue samples from the distal ileum were homogenized in RIPA lysis buffer containing $1 \%$ protease inhibitor (Beyotime, China), centrifuged at $10000 \mathrm{~g}$ for 10 minutes at $4^{\circ} \mathrm{C}$ and heated at $100^{\circ} \mathrm{C}$ for 10 minutes. Protein $(30 \mu \mathrm{g})$ was then loaded into a $10 \%$ SDS-PAGE gel for electrophoretic separation and transferred to a PVDF membrane. The membrane was blocked with $5 \%$ fat-free milk for $2 \mathrm{~h}$ and incubated with $1: 1000$ dilutions of antibodies against $\beta$-actin (Proteintech Group), claudin-1 (Abcam, 
USA), ZO-1 (Proteintech Group, USA), and occludin (Abcam, USA) overnight at $4^{\circ} \mathrm{C}$. The next day, the membrane was washed 4 times with Tris-buffered saline with Tween-20 (TBST) buffer and incubated with peroxidase-conjugated AffiniPure Goat Anti-Rabbit secondary antibody (Jackson ImmunoResearch, USA) for $90 \mathrm{~min}$ at room temperature. Protein bands were visualized with chemiluminescent HRP Substrate (Immobilon, USA). Protein concentration was measured using a BCA kit (YEASEN, China).

2.8. RT-PCR. Total RNA was isolated from the distal ileum using TRIzol ${ }^{\mathrm{TM}}$ (Invitrogen, USA). Reverse transcription was performed using the PrimeScript RT master mix kit (TaKaRa, Japan) and RT-PCR was performed using Premix $\mathrm{Taq}^{\mathrm{TM}}$ (TaKaRa, Japan). Gene expression was quantified using the 2- $\Delta \Delta \mathrm{Ct}$ method. Primer sequences against the lysozyme gene were as follows: forward $5^{\prime}$-AGGAATGGGAT GTCTGGCTAC- $3^{\prime}$ and reverse $5^{\prime}$-GGTATCCCACAGGC GTT CTT-3' (Sangon, China) [5]. $\beta$-Actin was used as an internal control. All experiments were performed in triplicate.

2.9. Immunofluorescence. Tissue sections were deparaffinized and rehydrated. Antigen retrieval was performed in citrate buffer, and endogenous peroxidase activity was blocked using $3 \%$ hydrogen peroxide in methanol for $20 \mathrm{~min}$. Sections were incubated with a primary antibody against lysozyme $(1: 1000$ dilution; DAKO) [4] at $4^{\circ} \mathrm{C}$ overnight. After washing, the sections were incubated with fluorescein-labeled secondary antibody (1:400 dilution; Servicebio, Wuhan, China) for $30 \mathrm{~min}$. Sections were stained with DAPI for $5 \mathrm{~min}$ to visualize nuclei.

2.10. 16s rRNA Sequencing and Analysis. Microbial genomic DNA was extracted from $500 \mathrm{mg}$ of feces from each rat using the $\mathrm{EZNA}^{\circledR}$ Soil DNA Kit (Omega BioTek, USA). DNA concentration was determined using a NanoDrop2000 (Thermo Scientific, USA). DNA integrity was verified by electrophoresis on a $1 \%$ agarose gel. The V3-V4 region of the bacterial 16S rRNA gene was amplified using the universal bacterial primers 338F (ACTCCTACGGGAGGCAGCA) and 806R (GGACTACHVGGGTWTCTAAT) (Sangon Biotech, China) [19]. PCR reactions were performed in a total volume of $20 \mu \mathrm{l}$ containing $10 \mathrm{ng}$ DNA template, $4 \mu \mathrm{l} \mathrm{FastPfu}$ buffer, $2 \mu \mathrm{l}$ dNTPs $(2.5 \mathrm{mM}), 0.8 \mu \mathrm{l}$ each primer $(5 \mu \mathrm{M})$, and $0.4 \mu \mathrm{l}$ of FastPfu polymerase (TransGen BioTech, Beijing, China). PCR cycles were as follows: $95^{\circ} \mathrm{C}$ for $3 \mathrm{~min}$, followed by 25 cycles at $95^{\circ} \mathrm{C}$ for $30 \mathrm{~s}, 55^{\circ} \mathrm{C}$ for $30 \mathrm{~s}$, and $72^{\circ} \mathrm{C}$ for $30 \mathrm{~s}$, and a final extension at $72^{\circ} \mathrm{C}$ for $5 \mathrm{~min}$. PCR products were analyzed by electrophoresis in a $2 \%$ agarose gel.

Equimolar concentrations of amplicons were sequenced on the Illumina MiSeq platform (Illumina, USA). Highquality sequences were assigned to the samples according to barcodes. A total of $3,450,879$ high-quality sequences and 1627 operational taxonomic units (OTUs) were obtained from 64 samples. OTUs were clustered at $97 \%$ nucleotide similarity level using UPARSE (version 7.1, http://drive5.com/uprase/) [20], and chimeric sequences were identified using UCHIME (http://www.drive5.com/ uchime/uchime_download.html) [21]. The taxonomy of each 16S rRNA gene sequence was analyzed using RDP Classifier2 (version 2.2, http://sourceforfe.net/projects/rdpclassifier/) against the SILVA 128 16S rRNA database (https://www.arb-silva.de/) [22, 23]. OTUs were used to calculate $\alpha$-diversity estimations, including diversity (Shannon, Simpson), richness (Ace, Chao-1, Sobs), and Good's coverage and rarefaction curve analysis using Mothur (version 1.30.2, http://www.mothur.org/wiki/Schloss_SOP\#Alpha_ diversity) [20]. Principal component analysis (PCA) was performed on OTUs by the Euclidean method, and a heatmap of gene expression was generated using the $\mathrm{R}$ gplot package (version 3.3.1). Analysis of linear discriminant analysis effect size (LEfSe) was performed to explore distinctive bacteria at various levels using a linear discriminant analysis (LDA) threshold of $>3.0$; the cladogram is displayed according to effective size. Comparative analysis between groups was performed using a nonparametric Wilcoxon test. A correlation heatmap was used to investigate correlations among SCFAs, intestinal barrier dysfunction, pathological injuries, TC, TG, lysozyme levels, and gut microflora. Phylogenetic investigation of communities by reconstruction of unobserved states (PICRUSt) was used to predict microbial functional features based on $16 \mathrm{~S}$ rRNA high-throughput sequencing data [24].

2.11. Analysis of Fecal SCFAs. Approximately $10 \mathrm{mg}$ of feces was mixed with $500 \mu \mathrm{l}$ methanol and $10 \mu \mathrm{l}$ internal standard (2-ethylbutyric acid) (Darmstadt, Germany). The mixture was ground up in a tissue grinder at $65 \mathrm{~Hz}$ for $120 \mathrm{~s}$, vortexed for $30 \mathrm{~s}$, and then centrifuged at $10000 \mathrm{~g}$ for $15 \mathrm{~min}$ at $4^{\circ} \mathrm{C}$. Supernatant $(400 \mu \mathrm{l})$ was then transferred to a $1.5 \mathrm{ml}$ tube, mixed with $50 \mu \mathrm{l}$ methanol, and centrifuged at $10000 \mathrm{~g}$ for $15 \mathrm{~min}$ at $4^{\circ} \mathrm{C}$. The supernatant was aspirated and analyzed by gas chromatography-mass spectrometry using an Agilent 6890A/5973C GC-MS platform (Agilent Technologies, USA) with a mass spectrometric range of $30-150(\mathrm{~m} / \mathrm{z})$ in a fullscan mode. Feature extraction and preprocessing were done using the XCMS package in R (version 2.13.2) [25], and then the data were normalized and edited into a two-dimensional data matrix in Microsoft Excel 2010.

2.12. Statistical Analysis. Data are presented as mean \pm standard deviation (SD). The Shapiro-Wilk test was used to test normality, and Levene's test was used to test homogeneity of variance. Comparisons between two groups were performed using the unpaired $T$-test (for normally distributed data) or Wilcoxon test (for skewed data). Spearman's rank correlation coefficient was used to test for correlations between the 50 most abundant bacterial species and biomarkers. For three or more groups, one-way ANOVA was preformed followed by Bonferroni's posttest for data with $F$ at $p<0.05$. All analyses were performed in SPSS 20.0 (IBM, Chicago, IL, USA). A $p$ value of $<0.05$ was considered statistically significant.

\section{Results}

3.1. Dithizone Depletes Paneth Cells and Lysozyme in the Ileum. A pilot experiment showed that the number of Paneth 

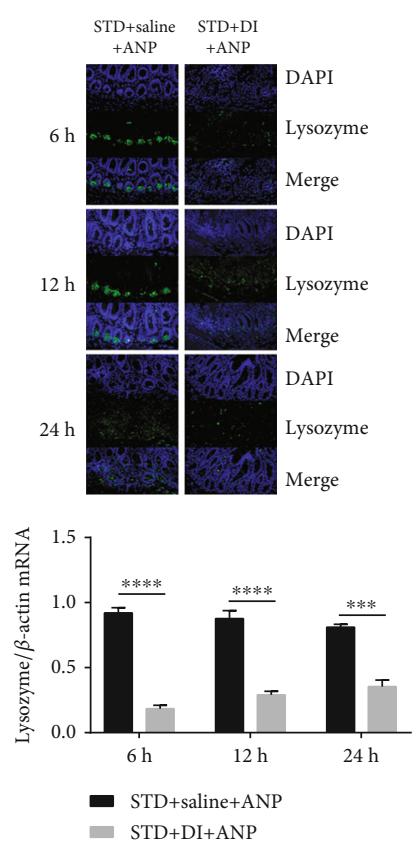

(a)
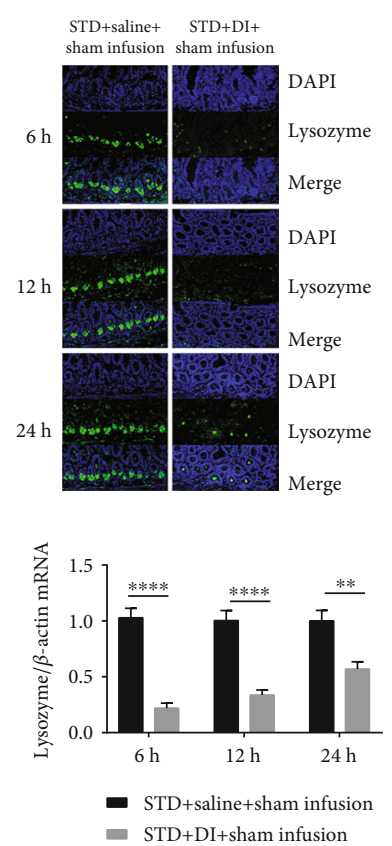

(b)
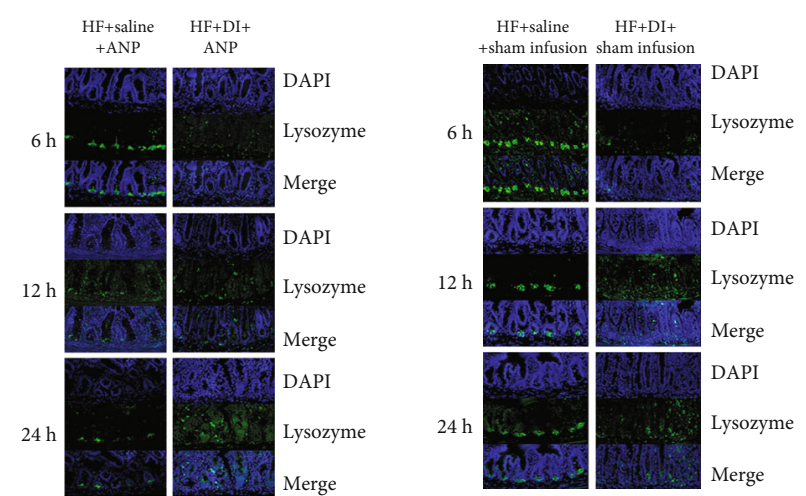

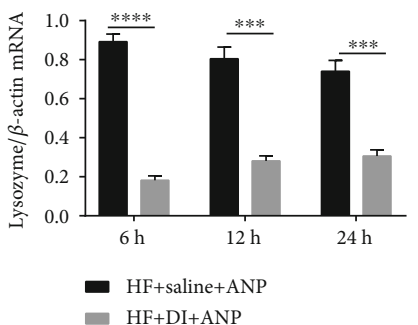

(c)

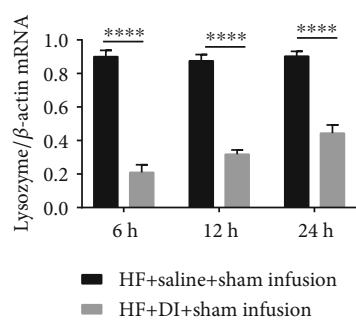

(d)

FIgURE 1: Dithizone depletes lysozyme in ileal crypts in rats. Lysozyme protein expression (green) in Paneth cells of the distal ileum as assessed by immunofluorescence. Nuclei were counterstained with DAPI (magnification $\times 200$ ). Lysozyme mRNA expression as assessed by RT-PCR. (a) STD+saline+ANP vs. STD+DI+ANP. (b) STD+saline+sham infusion vs. STD+DI+sham infusion. (c) HF+saline+ANP vs. $\mathrm{HF}+\mathrm{DI}+\mathrm{ANP}$. (d) HF+saline+sham infusion vs. HF+DI+sham infusion. ${ }^{*} p<0.05,{ }^{* *} p<0.01,{ }^{* * *} p<0.001,{ }^{* * * *} p<0.0001$, Student's $t$-test. ANP: acute necrotizing pancreatitis; DI: dithizone; HF: high-fat diet; STD: standard diet. Results were expressed as the mean \pm SD $(n=8)$.

cells was lowest at $6 \mathrm{~h}$ after dithizone treatment in rats on a standard or high-fat diet (Supplementary Figure 1). We therefore chose to perform retrograde sodium taurocholate infusion $6 \mathrm{~h}$ after dithizone treatment. At all time points, rats in all dietary groups treated with dithizone had lower lysozyme expression than those not treated with dithizone (Figure 1).

\subsection{High-Fat Diet Exacerbates Injuries Caused by Retrograde} Sodium Taurocholate Infusion. HF rats had higher levels of serum TG $(0.627 \pm 0.088$ vs. $0.202 \pm 0.048, p<0.001)$ and TC $(3.146 \pm 0.711$ vs. $1.414 \pm 0.272, p<0.001)$ than STD rats (Supplementary Figure 2A). Serum TNF- $\alpha$, IL- $1 \beta$, and IL$17 \mathrm{~A}$ levels and pancreatic injury scores were also higher in HF rats than STD rats at all time points assayed, suggesting exacerbation of ANP-associated pancreatic injury and systemic inflammation (Supplementary Figure 2C and D).

Compared to rats in the STD group, rats in the HF group had higher ileum injury scores $(6 \pm 0.707$ vs. $3.3 \pm 0.447$, $p<0.001$ ) (Supplementary Figure 2C and D), increased DAO activity (1332.2102 \pm 136.659 vs. $906.8835 \pm 66.453$, $p<0.001)$ and serum D-lactate $(6625.0142 \pm 501.387$ vs. $5903.5772 \pm 276.515, p=0.022$ ) (Supplementary Figure 2D), and decreased expression of lysozyme (Supplementary Figure 2B and E) and TJPs in the distal ileum (Figures 2(a) and 2(b) and Supplementary Figure 6) at $24 \mathrm{~h}$.

Rats in the HF group had lower Sobs, Ace, Chao-1, and Shannon indexes than rats in the STD group, suggesting lower microbiota diversity (Figure 2(c)). PCA also revealed that high-fat feeding induced alterations in the structure of intestinal microbiota, as demonstrated by clustering and separation of HF and STD samples (Figure 2(d)).

The most enriched predicted functional category of $16 \mathrm{~s}$ rRNA was carbohydrate transport and metabolism (Figure 2(e)). HF rats had lower levels of fecal SCFAs, including acetic acid, propionic acid, and butyric acid, than STD rats at 6 and $24 \mathrm{~h}$ (Figure $3(\mathrm{c})$ ).

3.3. Dithizone Exacerbates ANP-Related Injury, Inflammation, and Intestinal Permeability in Rats on Standard Diet. Rats in the DI+STD group had exacerbated pancreatic and ileal injuries (Figures 4(a) and 4(b)) and higher expression of serum inflammatory cytokines (Figure 4(c)) than rats in the STD group at all time points. DAO activity and serum D-lactate levels (Figure 4(d)) were also higher in $\mathrm{DI}+\mathrm{STD}$ rats than in STD rats, suggesting increased intestinal permeability. Expression of zo-1, occludin, and claudin-1 was decreased at $24 \mathrm{~h}$ (Figures 4(e) and 4(f) and Supplementary Figure 6). None of these parameters was significantly different between DI+STD and STD rats if ANP was not induced (Supplementary Figure 3A-D).

3.4. Dithizone Reduces Microbial Diversity in Rats with ANP on Standard Diet. Rats in the DI+STD group had lower Sobs, Ace, Chao-1, and Shannon indexes and a higher Simpson index than those in the STD group (Figure 5(a)), suggesting decreased microbiota diversity. PCA analysis showed that the microbiota composition in DI+STD and STD rats was 

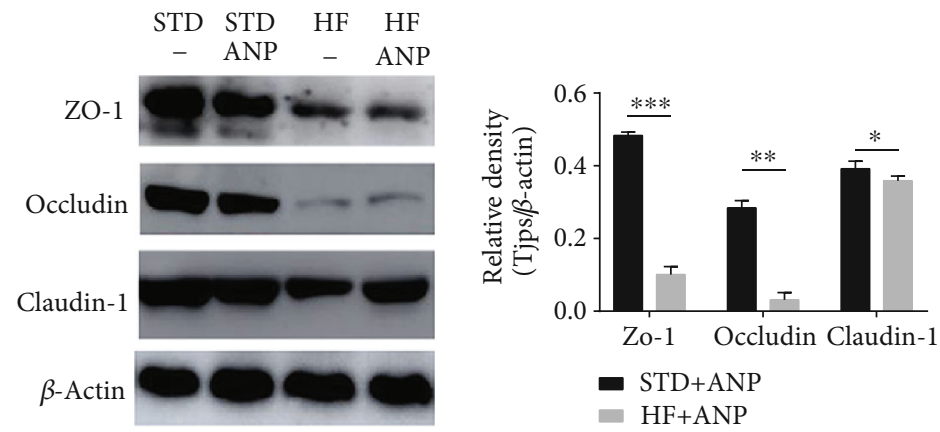

(a)
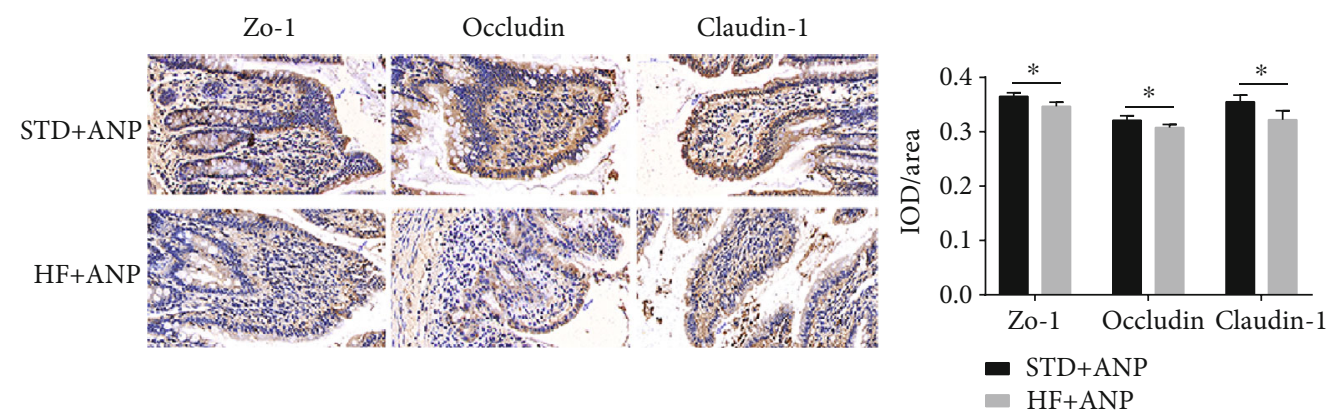

(b)
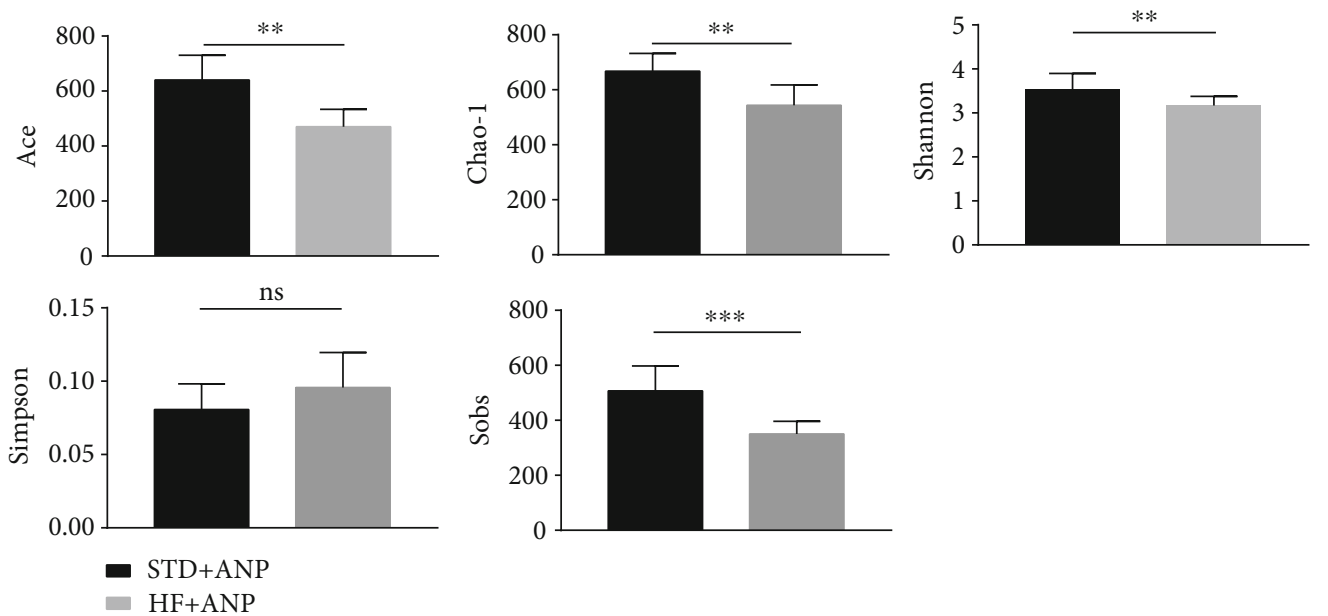

(c)

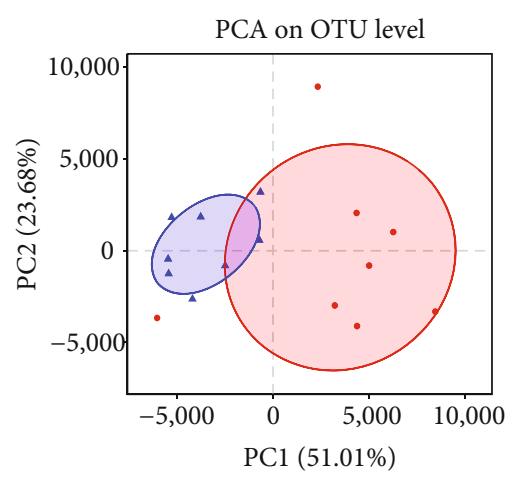

- STD+ANP

- $\mathrm{HF}+\mathrm{ANP}$

(d)

Figure 2: Continued. 


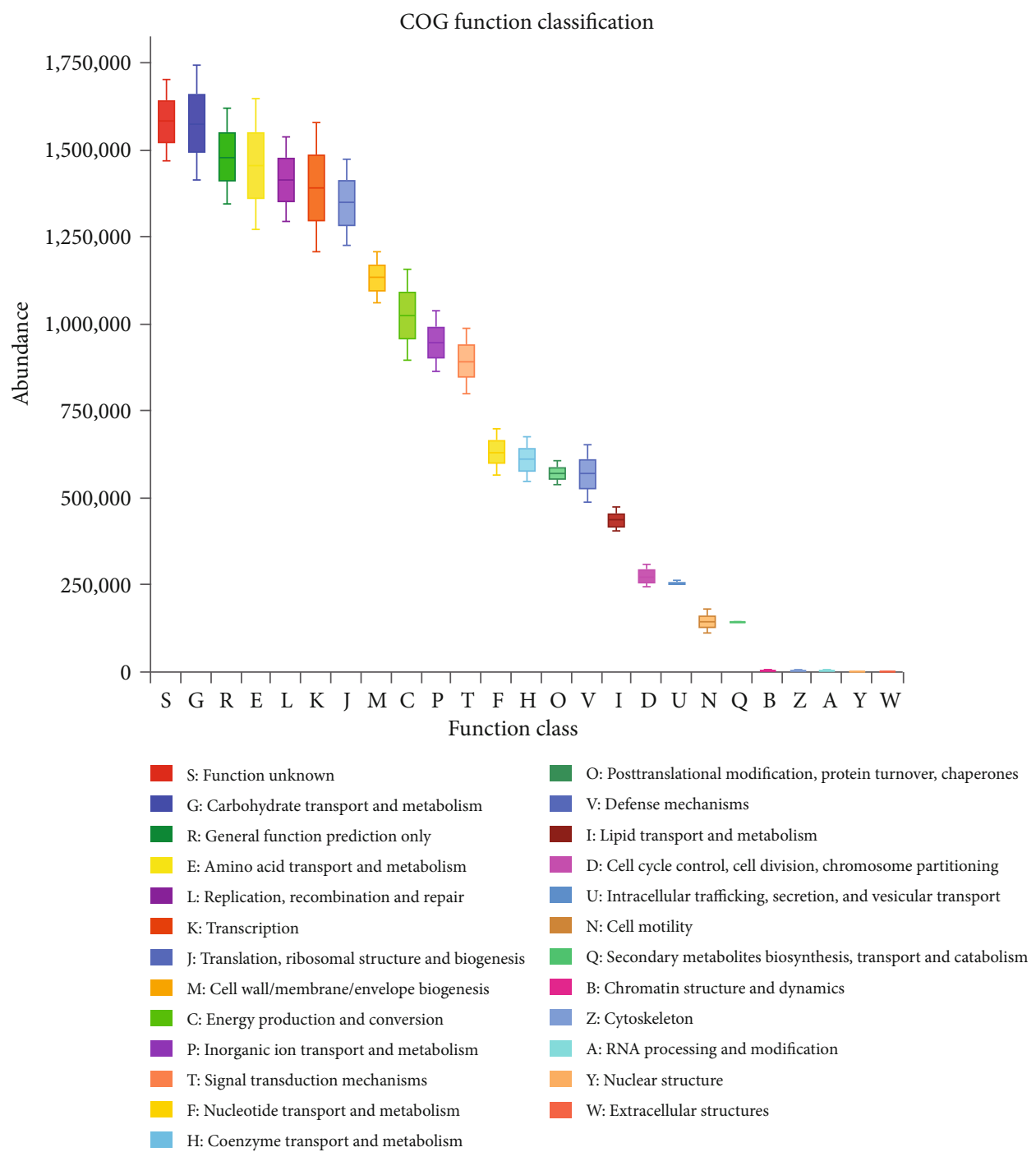

(e)

Figure 2: Effect of high-fat diet on ANP-associated injuries (TJPs and microbiota diversity) caused by retrograde sodium taurocholate infusion. Expression of caudin-1, occludin, and zo-1 as assessed by (a) western blot and (b) immunohistochemistry of the distal ileum. (c) Estimators of intestinal microbiota $\alpha$-diversity in rats fed a high-fat or standard diet $(n=8)$. (d) Principal component analysis of microbiota $\beta$-diversity $(n=8)$. (e) Predicted microbial functional categories of $16 \mathrm{~S}$ rRNA in fecal samples at $24 \mathrm{~h}$ after induction of ANP. ${ }^{*} p<0.05,{ }^{* *} p<0.01,{ }^{* * *} p<0.001$, Student's $t$-test.

distinct (Figure 5(b)). PC1 accounted for 59.75\% of total variance, while PC2 accounted for $14.93 \%$ of total variance.

The fecal microbial composition of DI+STD and STD rats is shown in Figures 5(c) and 5(d). Compared to rats in the STD group, rats in the DI+STD group showed enrichment of Gammaproteobacteria (from class to family), Cyanobacteria (from phylum to genus), Sutterella (genus), Actinobacteria (from phylum to class), Rothia (genus), Erysipelotrichia (from class to family), Allobaculum (genus), Globicatella (genus), Streptococcus (genus), and Enterococcus (genus) bacteria (Figure 6(c)). We also found significant enrichment of Epsilonproteobacteria (from class to genus), Deltaproteobacteria (from class to genus), Anaerovorax (genus), Bacilli (class), Rikenella (genus), and Lactobacillaceae (from family to genus) in STD rats compared to DI
+ STD rats. At the phylum level, DI+STD rats had fewer Bacteroidetes and more Actinobacteria than STD rats (Figure 6(a)). At the genus level, the following genera were lower in DI+STD rats than STD rats: Prevotellaceae NK3B31_group, Ruminiclostridium_9, Ruminococcus_1, Ruminiclostridium_5, Butyrivibrio, Roseburia, Helicobacter, Anaerotruncus, Oscillibacter, Ruminiclostridium_6, and Alloprevotella (Figure 6(a)). DI+STD rats also had more Allobaculum and Streptococcus than STD rats (Figure 6(a)). Rats in the DI+STD group had lower fecal SCFA levels than those in the STD group at 6 and $24 \mathrm{~h}$ (Figure 3(a)).

When ANP was not induced (sham induction), DI + STD rats showed a trend towards lower intestinal microbiota diversity than STD rats, but this was not statistically significant (Supplementary Figure 3E). PCA 


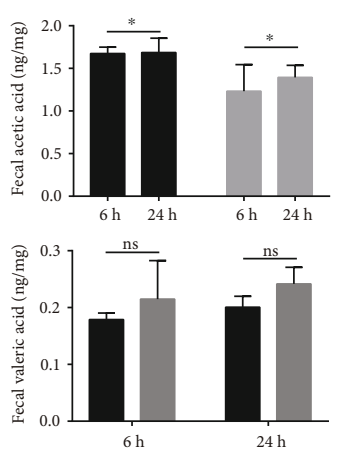

- STD+Saline+ANP

- STD+DI+ANP
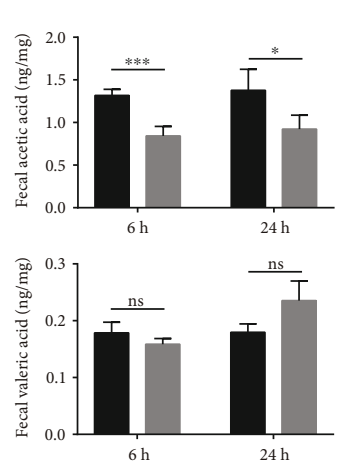

- $\mathrm{HF}+$ Saline+ANP

- HF+DI+ANP
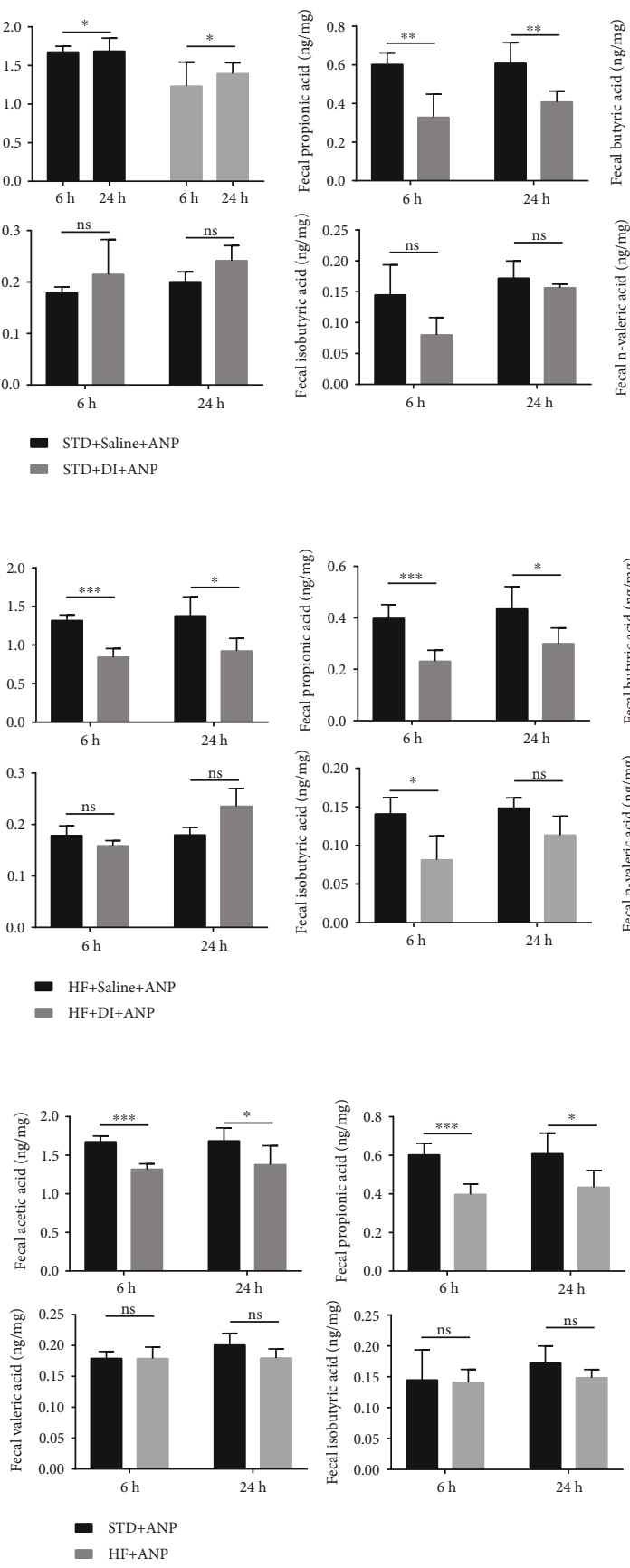
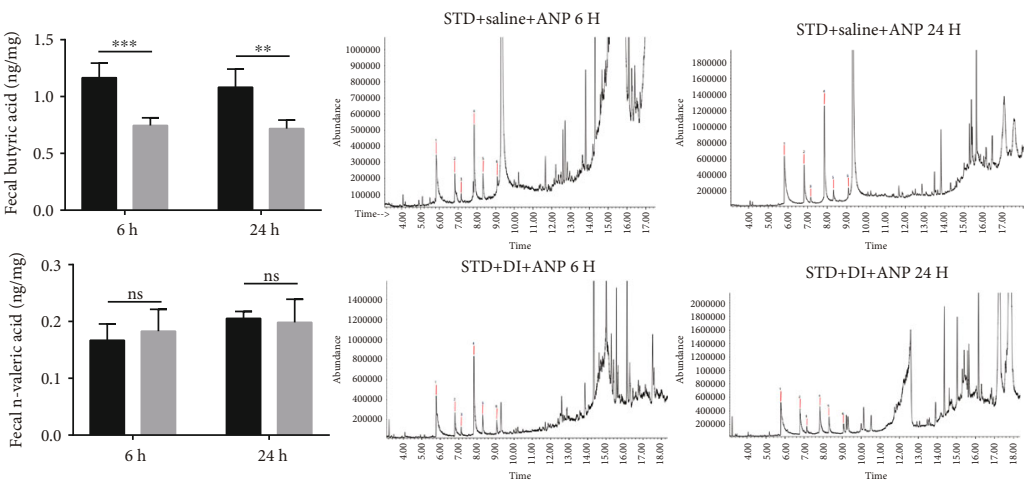

(a)
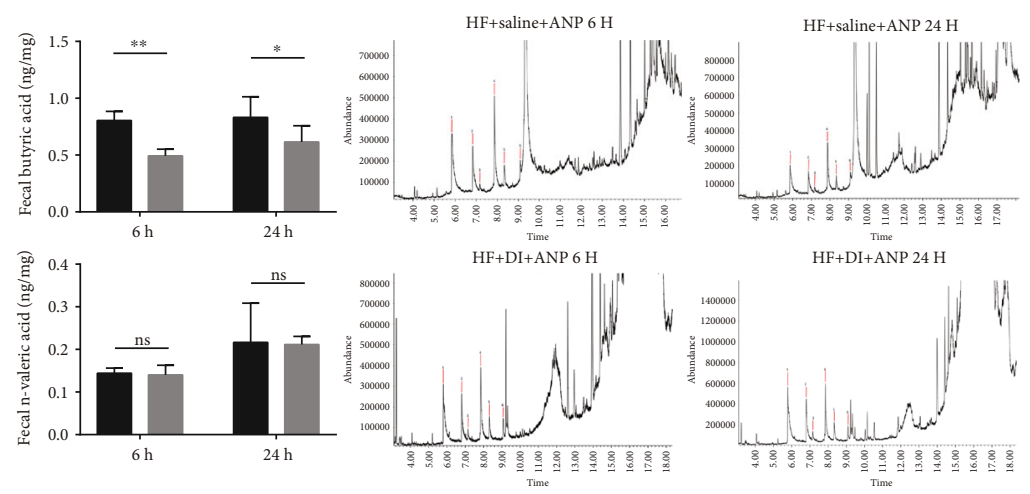

(b)
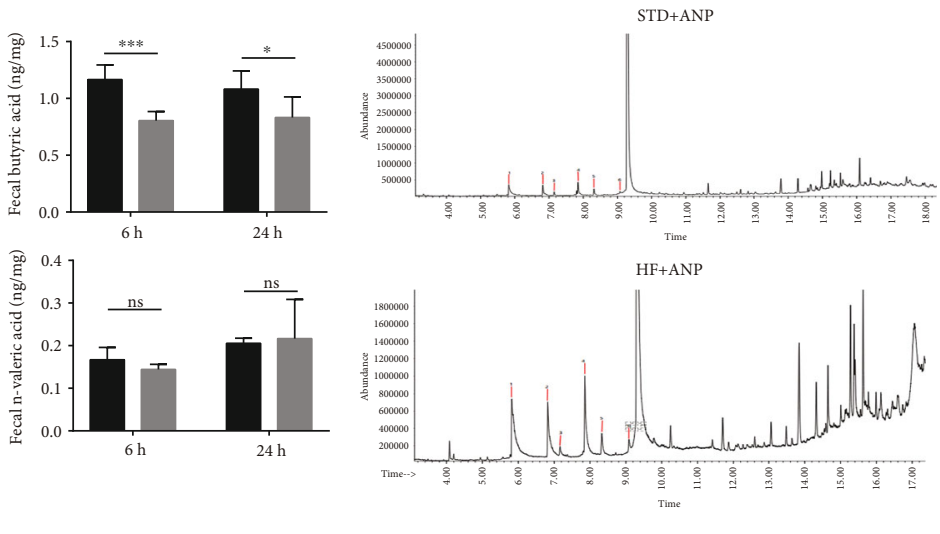

(c)

FIGURE 3: Fecal SCFAs levels as detected by gas chromatography-mass spectrometry. (a) Effects of dithizone on short-chain fatty acids in rats on standard diet followed by retrograde sodium taurocholate infusion. (b) Effects of dithizone on short-chain fatty acids in rats on high-fat diet followed by retrograde sodium taurocholate infusion. (c) Effects of high-fat diet on short-chain fatty acids in rats followed by retrograde sodium taurocholate infusion. ${ }^{*} p<0.05,{ }^{* *} p<0.01,{ }^{* * *} p<0.001$, Student's $t$-test. Results were expressed as the mean \pm SD $(n=5)$.

showed that DI+STD and STD rats still had distinct microbial compositions without induction of ANP (Supplementary Figure 3F). However, there was no significant difference in fecal SCFA levels at 6 and $24 \mathrm{~h}$ (Supplementary Figure 3G).

Spearman's rank correlation showed that Alloprevotella, Quinella, Roseburia, Oscillibacter, Ruminococcus_1, Anaerotruncus, and Ruminiclostridium_9 all positively correlated with fecal SCFAs and lysozyme expression, while they negatively correlated with serum DAO, serum D-lactate, serum inflammatory cytokines, and pathological injuries (Figure 6(b)). In contrast, Escherichia-Shigella and Enterococcus were negatively associated with SCFAs and lysozyme expression, while they positively associated with serum DAO, serum D-lactate, serum inflammatory cytokines, and pathological injuries (Figure 6(b)). 

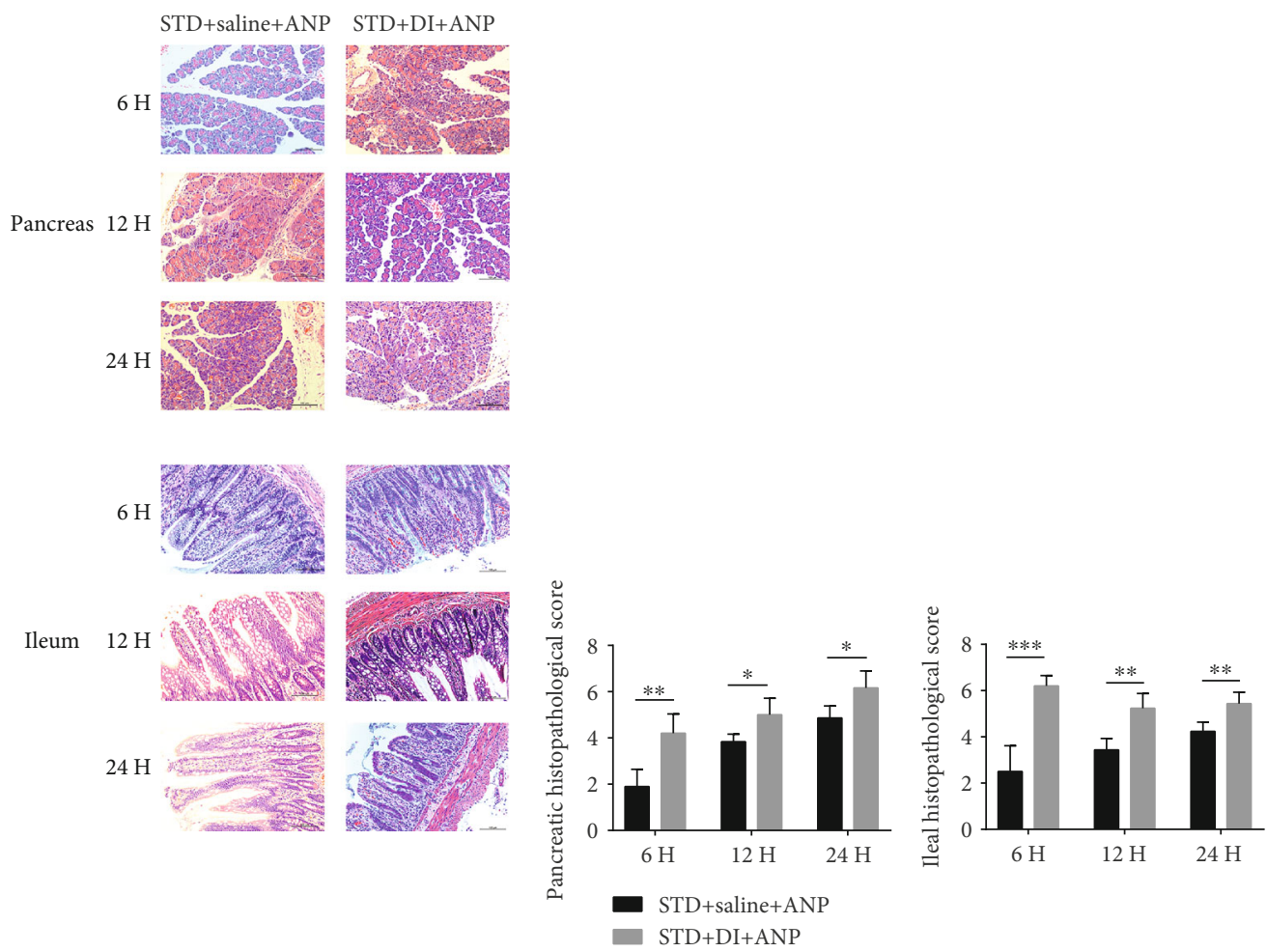

(a)

(b)
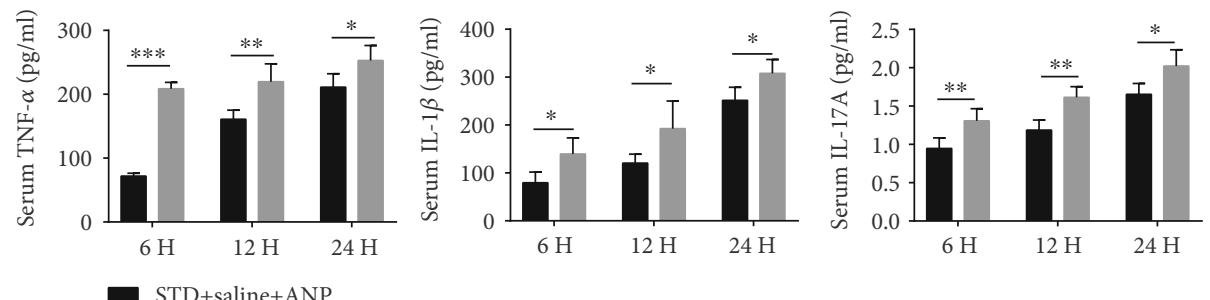

STD+DI+ANP

(c)

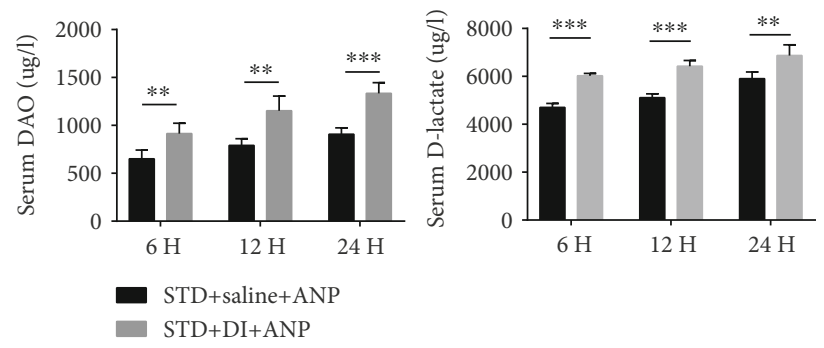

(d)

Figure 4: Continued. 

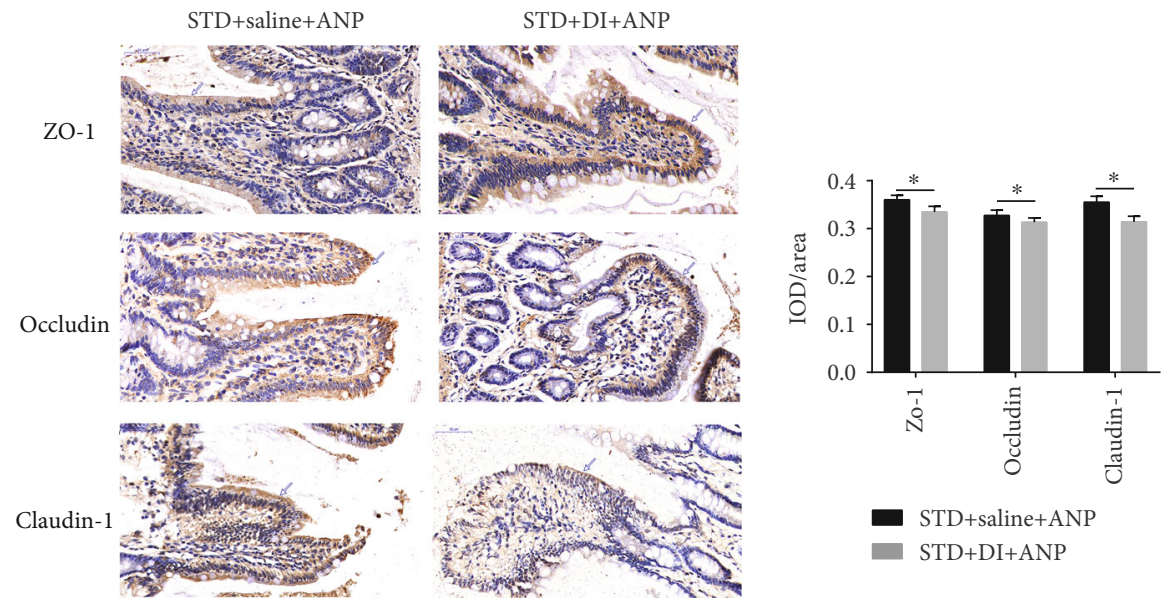

(e)

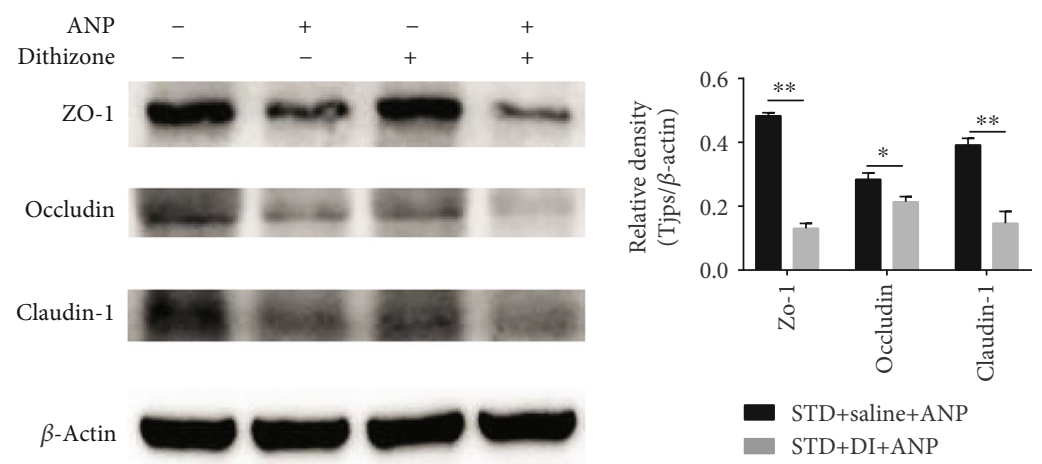

(f)

Figure 4: Effects of dithizone on pancreatic and intestinal injuries in rats on standard diet followed by retrograde sodium taurocholate infusion. (a) Pancreas and distal ileum sections of rats treated with dithizone or saline. Tissue was stained with H\&E (magnification $\times 200$ ). (b) Histopathological scores of the pancreas and distal ileum. (c) Serum TNF $\alpha$, IL- $1 \beta$, supplementary, and IL-17A levels in rats treated with dithizone or saline. (d) DAO activity and serum D-lactate in rats treated with dithizone or saline. (e) Distal ileum sections showing immunostaining for claudin-1, occludin, and zo-1, together with quantification of claudin-1, occludin, and zo-1 staining. (f) Expression of claudin-1, occludin, and zo-1 as assessed by western blot. ${ }^{*} p<0.05,{ }^{* *} p<0.01,{ }^{* * *} p<0.001$, Student's $t$-test. Results were expressed as the mean $\pm \mathrm{SD}(n=5)$.

3.5. Dithizone Exacerbates ANP-Related Injuries, Inflammation, and Intestinal Permeability in Rats on HighFat Diet. Rats in the DI+HF group had higher pancreatic injury scores (Figures $7(\mathrm{a})$ and $7(\mathrm{~b})$ ) and higher serum levels of TNF- $\alpha$, IL- $1 \beta$, and IL-17A (Figure 7(c)) than rats in the $\mathrm{HF}$ group at all time points. DI+HF rats also had higher ileal injury scores (Figures 7(a) and 7(b)), higher DAO activity and serum D-lactate (Figure 7(d)), and decreased zo-1 and claudin-1 expression at $24 \mathrm{~h}$ (Figures $7(\mathrm{e})$ and $7(\mathrm{f})$ and Supplementary Figure 6). There was no difference in any of these parameters between DI+HF and HF rats if ANP was not induced (Supplementary Figure 4A-D).

3.6. Dithizone Reduces Microbial Diversity in Rats with ANP on High-Fat Diet. DI+HF rats had lower Sobs, Ace, Chao-1, and Shannon indexes and a higher Simpson index than HF rats, suggesting reduced microbiota diversity (Figure 8(a)). PCA analysis showed that the fecal microbial composition of DI+HF and $\mathrm{HF}$ rats was distinct (Figure 8(b)). PC1 accounted for $44.92 \%$ of total variance, and PC2 accounted for $35.79 \%$ of total variance.
Figures $8(\mathrm{c})$ and $8(\mathrm{~d})$ show the fecal microbial composition in DI+HF and HF rats. Compared to the HF group, rats in the DI+HF group had enrichment of Firmicutes (from phylum to genus), Peptococcaceae (family), Allisonella (genus), Adlercreutzia (genus), and Dorea (genus) (Figure 9(c)). There was also enrichment of Proteobacteria (from phylum to family) and Escherichia_shigella (genus) in HF rats compared to DI+HF rats. At the phylum level, DI+HF rats had more Spirochaetae than HF rats (Figure 9(a)). The following genera had lower enrichment in DI+HF rats than HF rats: Dorea, Anaerostipes, Tyzzerella, Oscillibacter, Bilophila, Parasutterella, Acetatifactor, Lachnospriraceae_NK4A136_group, Prevotellaceae_NK3B31_ group, Ruminiclostridium_9, Ruminococcus_1, Roseburia, and Alloprevotella (Figure 9(a)). In contrast, Blautia, Candidatus_stoquefichus, Treponena_2, and Enterococcus were higher in DI+HF rats than HF rats (Figure 9(a)). DI+HF rats also had lower levels of fecal SCFAs than HF rats at 6 and $24 \mathrm{~h}$ (Figure 3(b)).

When ANP was not induced (sham induction), DI+HF rats showed a trend towards lower intestinal microbial 

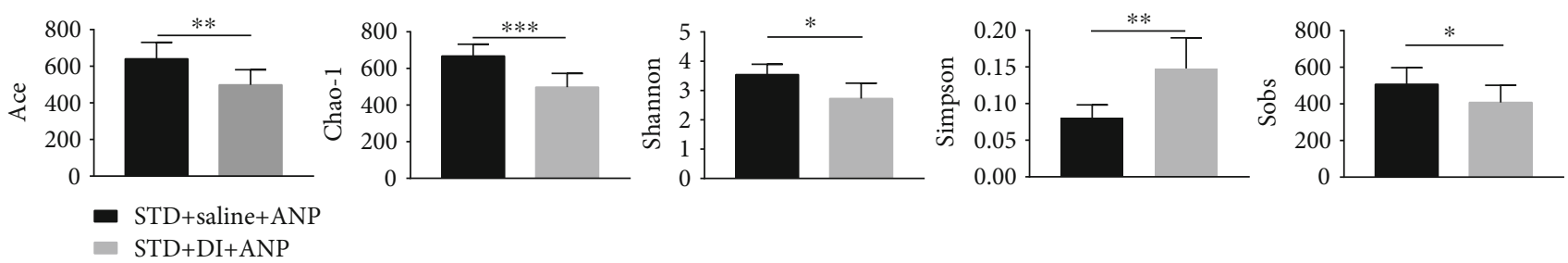

- STD+DI+ANP

(a)

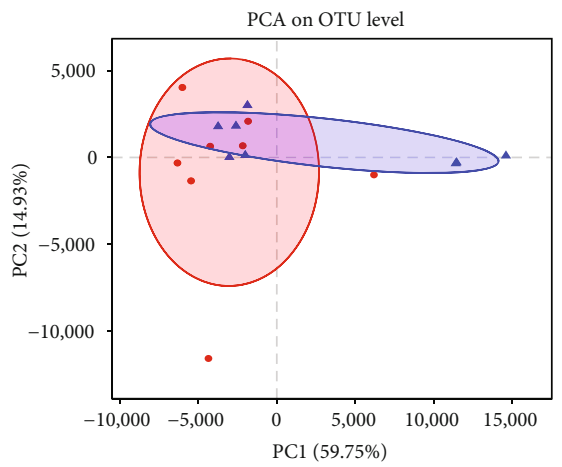

- STD+saline+ANP

- STD+DI+ANP

(b)

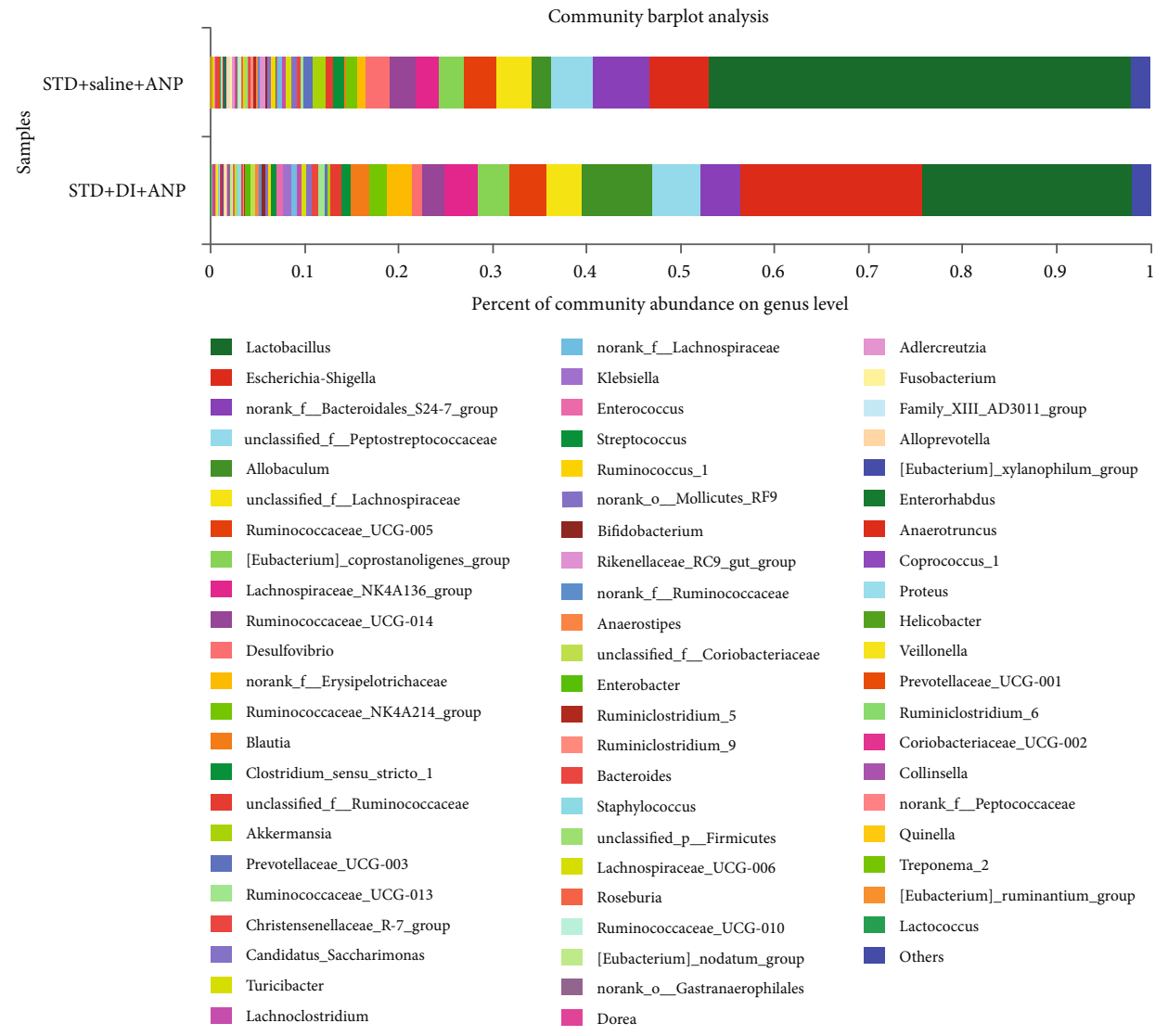

(c)

Figure 5: Continued. 


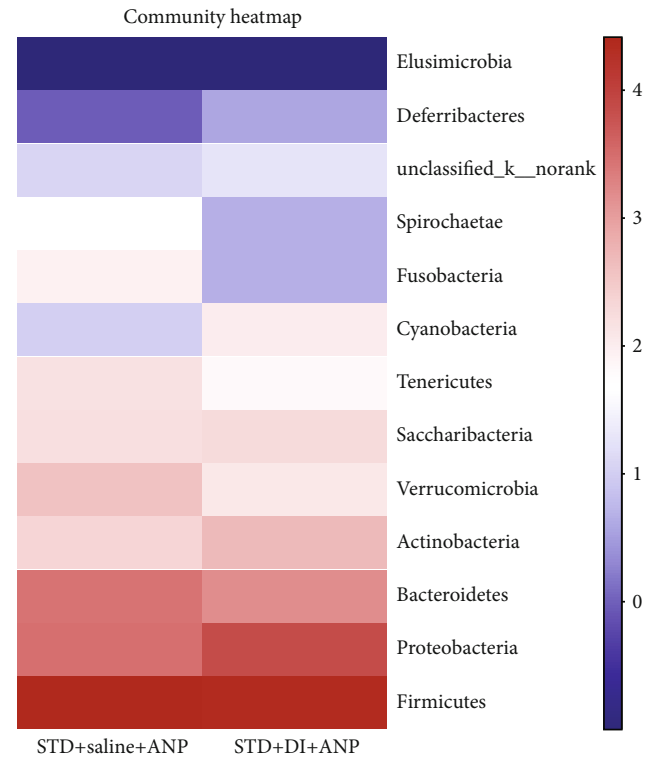

(d)

FIGURE 5: Effects of dithizone on diversity of fecal microbiota in rats on standard diet followed by retrograde sodium taurocholate infusion. (a) Estimators of intestinal microbiota $\alpha$-diversity in rats treated with dithizone or saline. (b) Principal component analysis of intestinal microbiota $\beta$-diversity in rats treated with dithizone or saline. (c) Relative abundance of bacterial genera in rats treated with dithizone or saline. (d) Relative abundance heatmap of bacterial phyla in intestinal microbiota of rats treated with dithizone or saline. ${ }^{*} p<0.05,{ }^{* *} p<$ $0.01,{ }^{* * *} p<0.001$, Student's $t$-test. $n=8$ for each group.

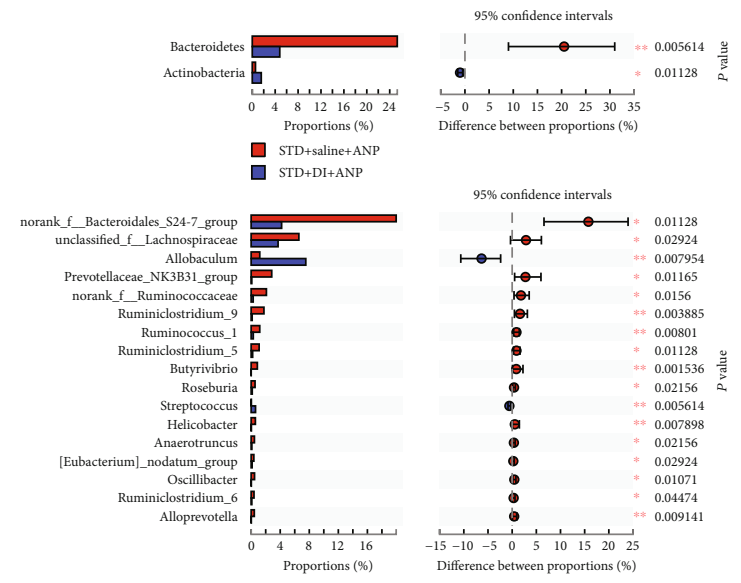

(a)

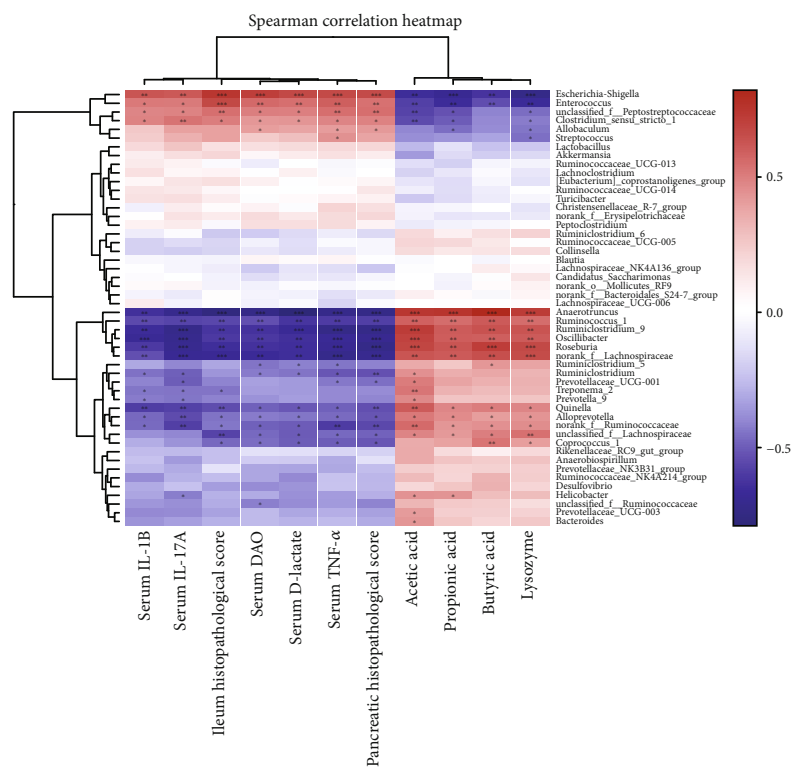

(b)

Figure 6: Continued. 


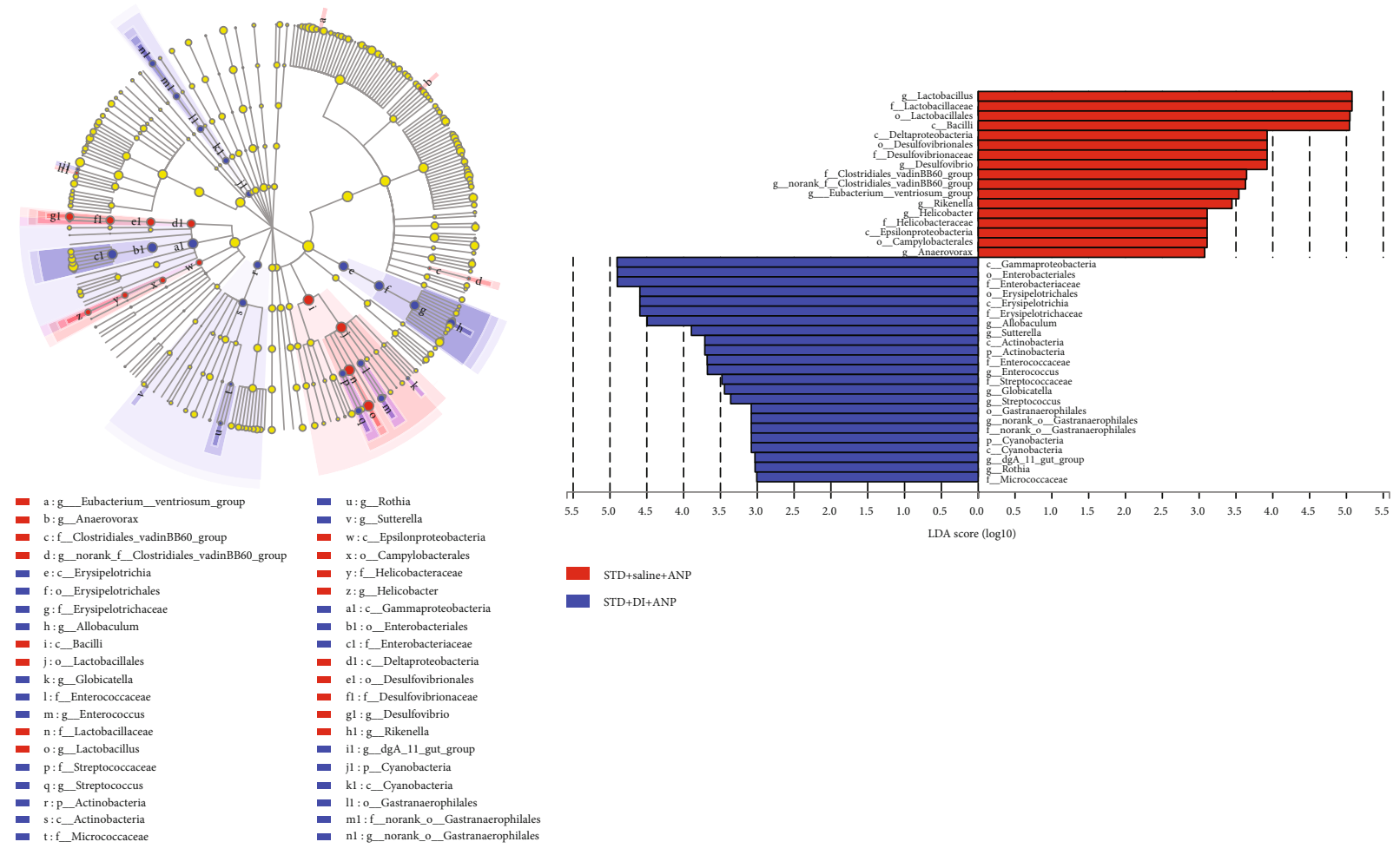

(c)

FIgURE 6: Effects of dithizone on microbiota composition in rats on standard diet followed by retrograde sodium taurocholate infusion. (a) Bacterial phyla and genera with significantly different abundance in rats treated with dithizone or saline. ${ }^{*} p<0.05,{ }^{* *} p<0.01,{ }^{* * *} p<0.001$, Wilcoxon test. (b) Heatmap showing correlation among SCFAs, lysozyme expression, intestinal barrier dysfunction, serum lipid, pathological changes, and gut microflora. Blue indicates a negative correlation, while red indicates a positive correlation. ${ }^{*} p<0.05,{ }^{* *} p<0.01,{ }^{* * *} p<0.001$ , Spearman test. (c) Analysis of linear discriminant analysis effect size (LEfSe) using multilevel species hierarchy tree and LEfSe bars determined by linear discriminant analysis (LDA). $n=8$ for each group.

diversity than HF rats, but this was not statistically significant (Supplementary Figure 4E). PCA analysis showed that DI+HF and $\mathrm{HF}$ rats had different microbial compositions even without ANP induction (Supplementary Figure 4F). However, there was no significant difference in fecal SCFAs at 6 and $24 \mathrm{~h}$ except for n-valeric acid (Supplementary Figure 4G).

Spearman's rank correlation showed that Desulfovibrio, Alloprevotella, Oscillibacter, Quinella, Bacteroides, Lachnospiraceae_NK4A136_group, Prevotellaceae_NK3B31_group, Ruminococcus_1, and Ruminiclostridium_9 all positively correlated with SCFAs and lysozyme expression, while they negatively correlated with TC, TG, DAO activity, serum Dlactate, serum inflammatory cytokines, and pathological injuries (Figure 9(b)). In contrast, Allobaculum, Bifidobacterium, Escherichia-Shigella, Fusobacterium, and Peptoclostridium negatively correlated with SCFAs and lysozyme expression, while they positively correlated with TC, TG, DAO activity, serum D-lactate, serum inflammatory cytokines, and pathological injuries (Figure 9(b)).

\section{Discussion}

In this study, we show that depleting Paneth cells using dithizone exacerbates pancreatic pathology, inflammation, and intestinal injury in rat models of ANP after normal and high-fat diet.
High-fat feeding exacerbated pancreatic and intestinal ANP-associated injuries. This is consistent with previous studies showing that hypertriglyceridemia is a crucial factor that exacerbates pancreatic pathology and intestinal injuries in ANP $[5,16,26]$. Several studies have demonstrated that over 8-week high-fat diet decreased tight-junction proteins and increased intestinal permeability in mice [14, 27, 28]. In our current study, we found 2-week high-fat diet decreased $\mathrm{TJ}$ proteins and increased intestinal permeability in ANP rats, which was consistent with our previous study [5]. However, the mechanism is not clear. High-fat feeding also reduced fecal microbial diversity, in agreement with the findings of previous studies $[11,29]$. These observations are likely linked to the decrease in Paneth cell number and lysozyme expression in response to high-fat feeding. Lysozyme is an essential effector secreted by Paneth cells and its expression is known to be correlated with microbiota homeostasis [30].

Paneth cell ablation with dithizone aggravated pancreatic and intestinal injuries in rats with ANP on standard or high-fat diets. Paneth cell depletion in mice has been reported to cause intestinal barrier damage and enhanced pathogen transmission [31]. Translocation of bacteria from the small bowel [32] can aggravate damage to the intestinal barrier in ANP [33], and intestinal barrier dysfunction is a major contributor to ANP complications [34]. In contrast, Paneth cell depletion alone (without ANP 


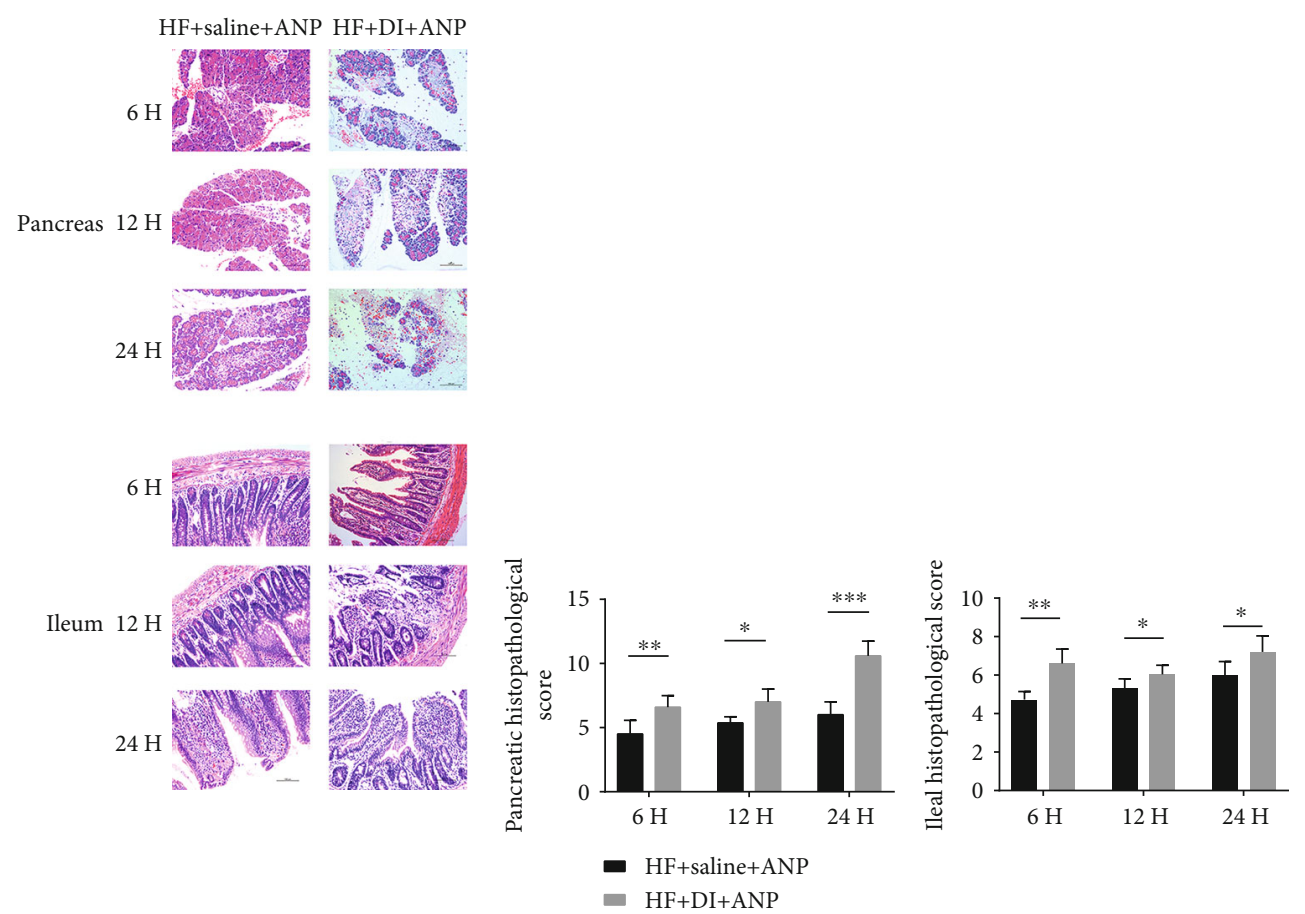

(a)

(b)
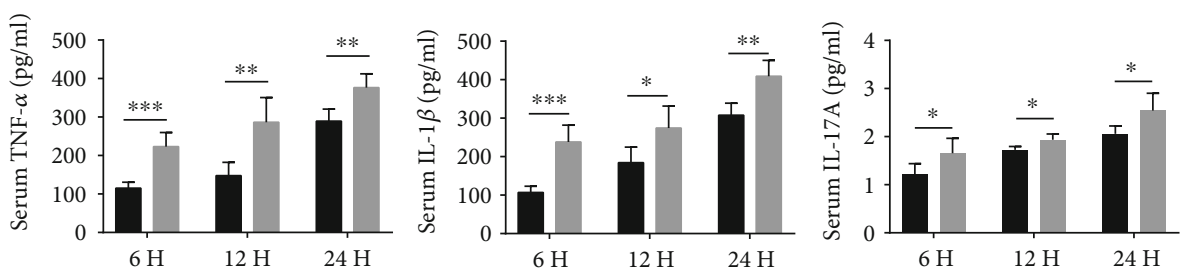

- $\mathrm{HF}+$ saline+ANP

- HF+DI+ANP

(c)

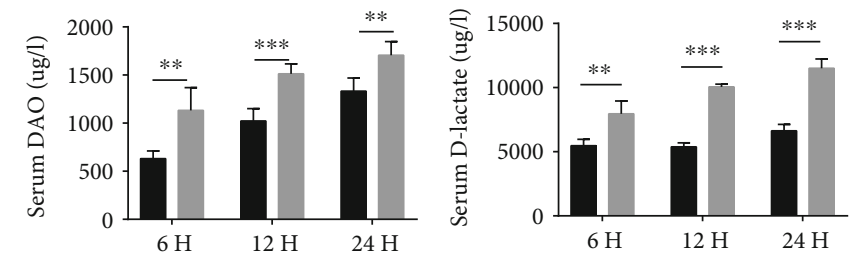

- HF+saline+ANP

- HF+DI+ANP

(d)
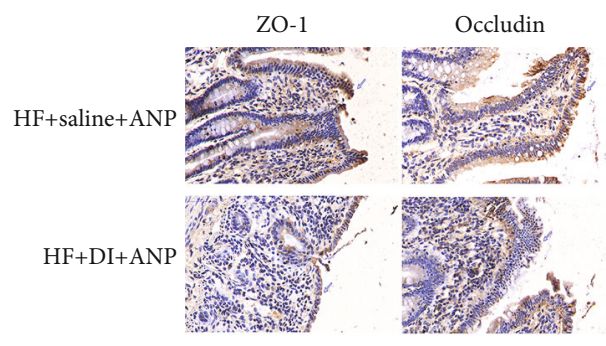

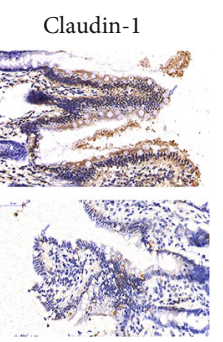

(e)

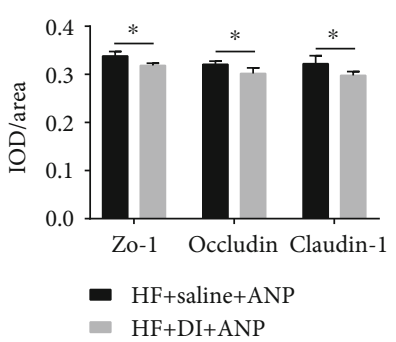

- HF+DI+ANP

FIgURe 7: Continued. 


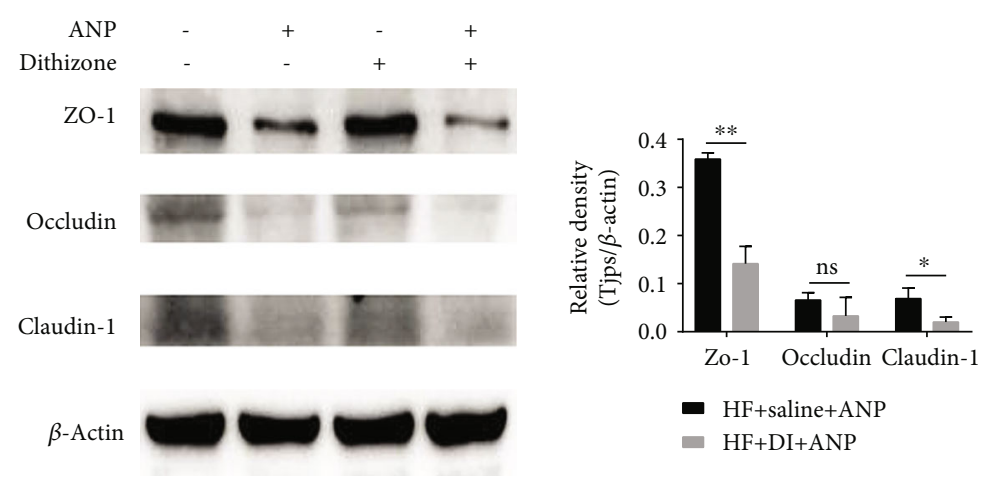

(f)

FIgURE 7: Effects of dithizone on pancreatic and intestinal injuries in rats on high-fat diet followed by retrograde sodium taurocholate infusion. (a) Sections of the pancreas and the distal ileum stained with H\&E in rats treated with dithizone or saline (magnification $\times 200$ ). (b) Histopathological scores of the pancreas and distal ileum. (c) Serum inflammatory cytokines in rats treated with dithizone or saline. (d) DAO activity and serum D-lactate in rats treated with dithizone or saline. (e) Sections of distal ileum showing immunostaining for claudin-1, occludin, and zo-1 in rats treated with dithizone or saline, together with quantification of claudin-1, occludin, and zo-1 immunostaining. (f) Expression of intestinal epithelial TJPs as assessed by western blot. ${ }^{*} p<0.05,{ }^{* *} p<0.01,{ }^{* * *} p<0.001$, Student's $t$ -test. Results were expressed as the mean \pm SD $(n=5)$.

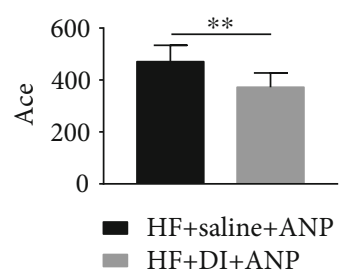

- HF+DI+ANP
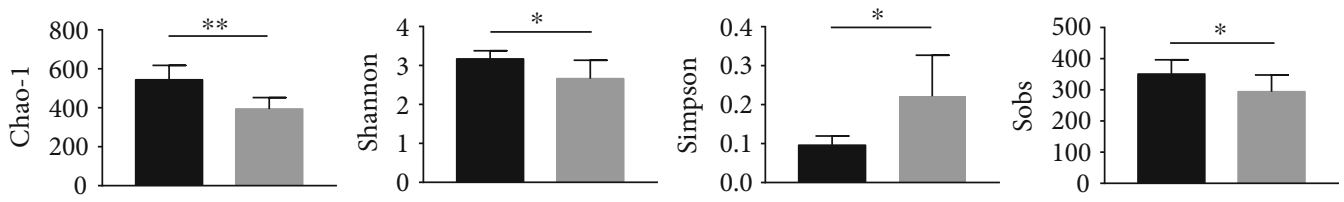

(a)

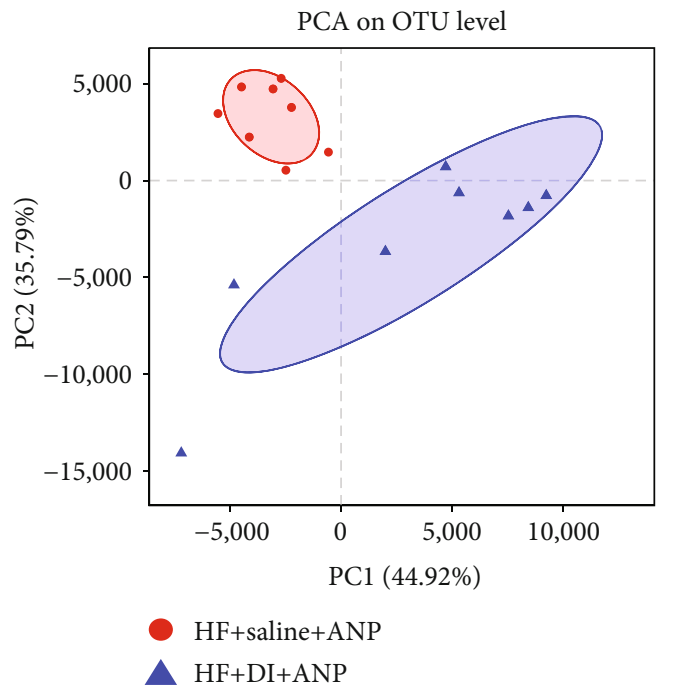

(b)

Figure 8: Continued. 


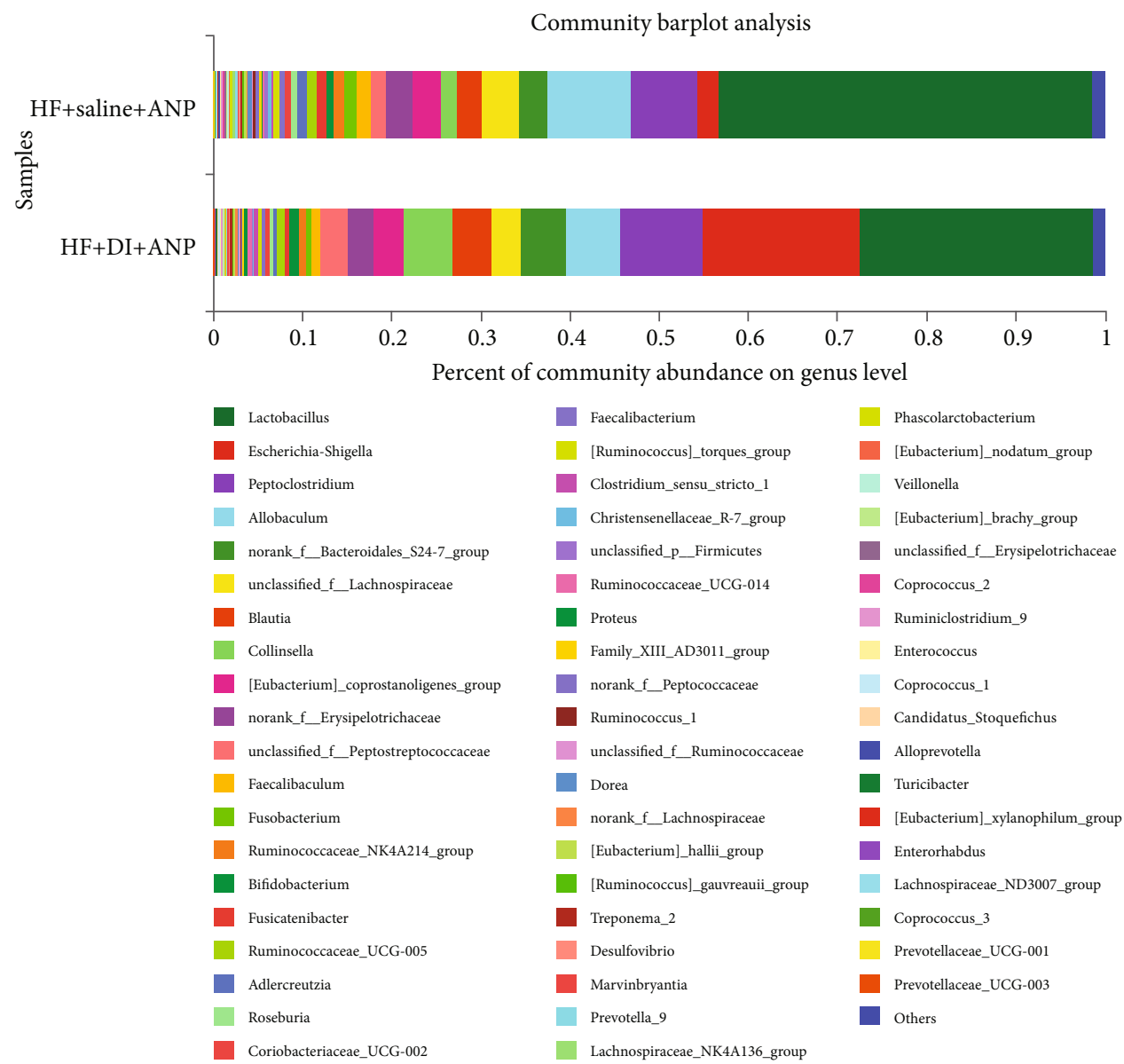

(c)

Community heatmap

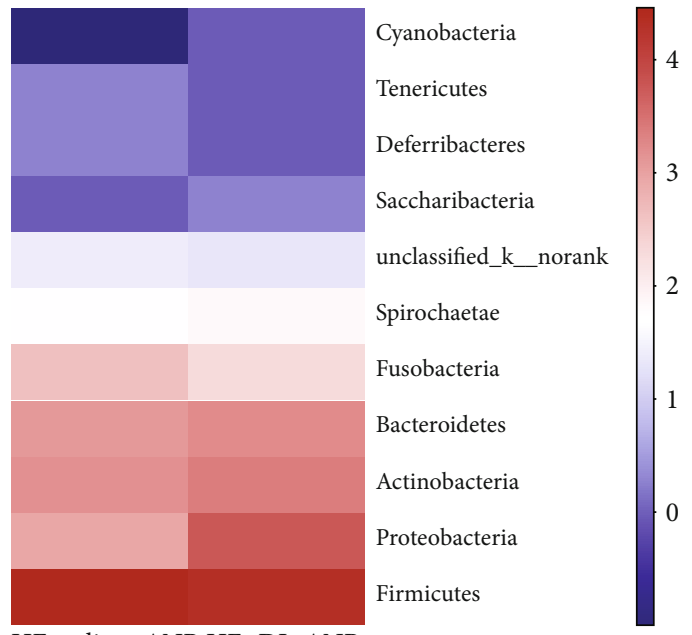

$\mathrm{HF}+$ saline+ANP HF+DI+ANP

(d)

FiguRE 8: Effects of dithizone on microbiota diversity in rats on high-fat diet followed by retrograde sodium taurocholate infusion. (a) Estimators of intestinal microbiota $\alpha$-diversity in rats treated with dithizone or saline. (b) PCA of microbial $\beta$-diversity in rats treated with dithizone or saline. (c) Relative abundance of intestinal microbial genera in rats treated with dithizone or saline. (d) Heatmap of relative abundance of bacterial phyla in intestinal microbiota of rats treated with dithizone or saline. ${ }^{*} p<0.05,{ }^{* *} p<0.01,{ }^{* * *} p<0.001$, Student's $t$ -test. $n=8$ for each group. 


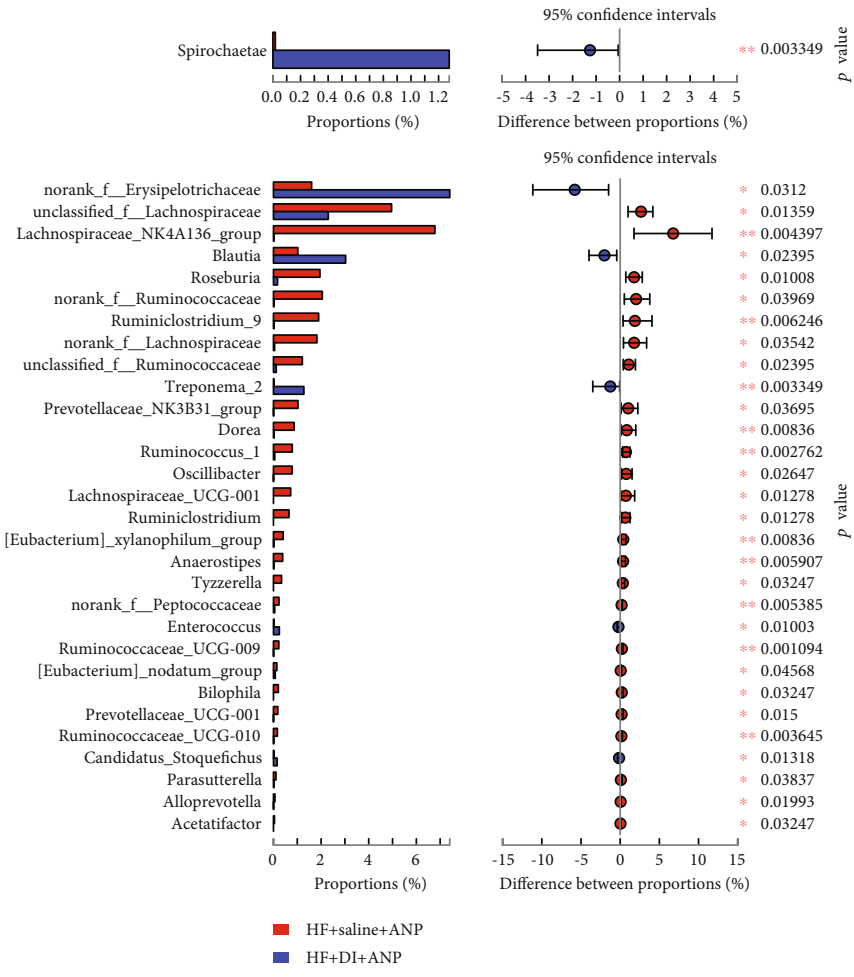

(a)
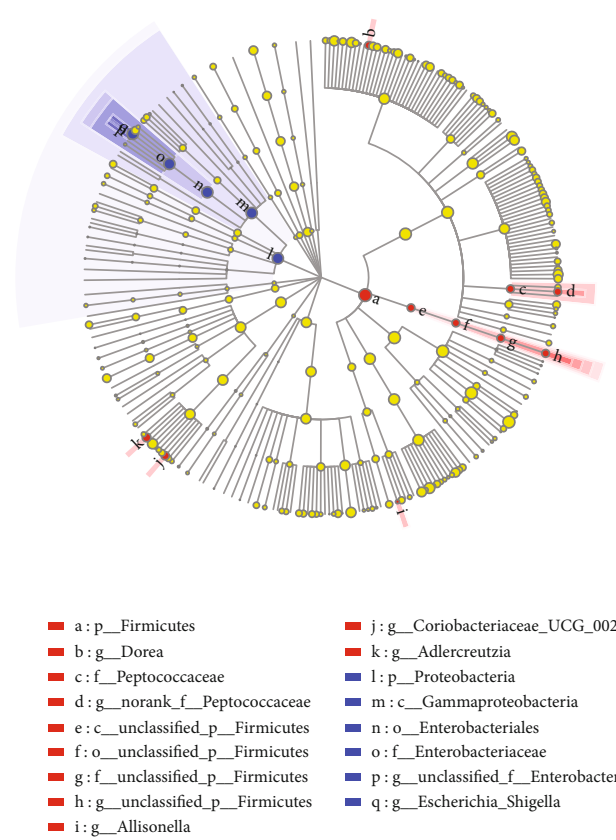

- j:g_Coriobacteriaceae_UCG_002

- k:g_Adlercreutzi

- 1:p_Proteobacteria

- $\mathrm{m}: \mathrm{c} \_$Gammaproteobacteri

- $\mathrm{n}: \mathrm{o} \_$Enterobacteriales

- o:f_Enterobacteriaceae

- p:g_unclassified_f_Enterobacteriaceae

- q:g_Escherichia_Shigella

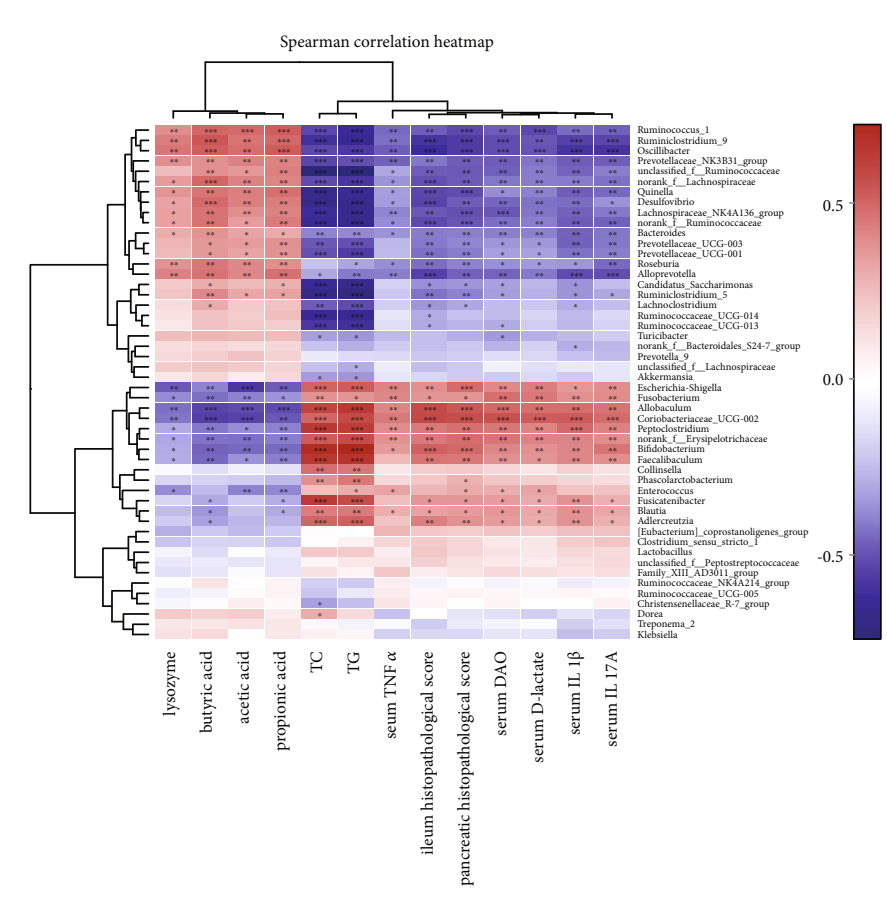

(b)

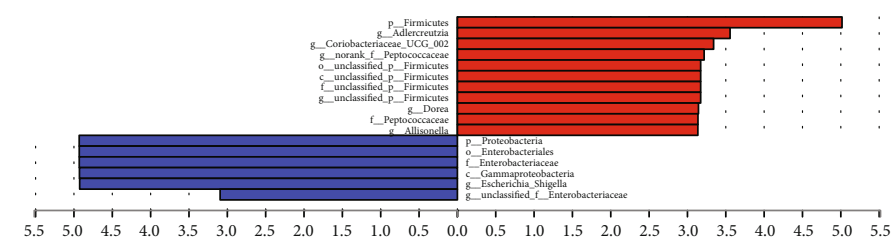

- HF+saline+ANP

- $\mathrm{HF}+\mathrm{DI}+\mathrm{ANP}$

(c)

FIGURE 9: Effects of dithizone on microbiota composition in rats on high-fat diet followed by retrograde sodium taurocholate infusion. (a) Bacterial phyla and genera with significantly different abundance in rats treated with dithizone or saline. ${ }^{*} p<0.05,{ }^{* *} p<0.01$, ${ }^{* * *} p<0.001$, Wilcoxon test. (b) Heatmap showing correlation among SCFAs, lysozyme, intestinal barrier dysfunction, serum lipid, pathological changes, and gut microflora. Blue indicates a negative correlation, while red indicates a positive correlation. ${ }^{*} p<0.05$, ${ }^{* *} p<0.01,{ }^{* * *} p<0.001$. Spearman test. (c) LEfSe multilevel species hierarchy tree and LEfSe bars discriminated by LDA. $n=8$ for each group. 
induction) had no significant effect on pancreatic and intestinal injuries or intestinal microbiota composition. This is consistent with previous studies showing that depletion of Paneth cells with dithizone does not affect the intestinal barrier [35].

Paneth cell depletion also changed intestinal microbiota structure and decreased microbial diversity in rats with ANP after normal and high-fat diet. Paneth cells directly sense commensal microbiota through a TLR-MyD88dependent pathway [36] and regulate innate immunity in the intestine [7]. Intestinal microbiota is an irreplaceable factor in maintaining intestinal homeostasis [37]. Probiotics can prevent DSS-induced intestinal injuries by strengthening intestinal barrier integrity [38]. A recent study shows that interactions between diet and intestinal microbiota can modify intestinal permeability and colitis severity in mice [39].

Analysis of the microbial phylogenetic composition showed that Bacteroidetes was decreased and Actinobacteria was increased in rats on standard diet, while Spirochaetae was increased in rats on high-fat diet. Bacteroidetes is associated with high-fat feeding and chronic respiratory inflammation [40, 41]. Several bacterial genera including Alloprevotella, Oscillibacter, Quinella, Ruminococcus_1, Ruminiclostridium_9, and Escherichia-Shigella also correlated with increased intestinal permeability, inflammation, and pancreatic and ileal injury in rats fed standard or highfat diets. Alloprevotella has been reported to negatively correlate with inflammation and positively correlate with HDL-C in rats with type 2 diabetes [42], while Allobaculum negatively correlates with serum alanine aminotransferase in mice with nonalcoholic fatty liver disease [43].

Paneth cell depletion decreased fecal SCFAs in ANP rats on standard or high-fat diets. Several bacterial genera were also correlated with SCFAs, in agreement with the findings of a previous study [42]. SCFAs are known to regulate intestinal barrier integrity and inflammatory responses through GPR41 and GPR43 [44]. Previous studies have reported that SCFAs act as histone deacetylase inhibitors to suppress the inflammatory response, NLRP3 inflammasome activity and autophagy [45]. We speculate that several bacterial genera may exert anti-inflammatory effects by regulating SCFAs.

Paneth cell ablation aggravates pancreatic and intestinal injuries after normal and high-fat diet. Meanwhile, intestinal microbiota and its metabolites are greatly changed. As shown in Supplementary Figure 5, we hypothesized that the mechanism by which Paneth cell ablation aggravates ANP after normal or high-fat diet may be related to intestinal barrier dysfunction, proinflammatory cytokines, microbiota dysbiosis, and short-chain fatty acids. However, its mechanism is still unclear. We will explore the role of Paneth cells in ANP in the further studies.

\section{Conclusions}

Paneth cell depletion enhances pancreatic and intestinal injuries in a rat model of ANP on standard or high-fat diets. Changes in microbiota and SCFAs are associated with pancreatic and intestinal injury. Our study sheds further light on the associations among Paneth cells, intestinal microbiota, and ANP. SCFAs produced by microbiota may have potential as new therapeutic targets for treating ANP.

\section{Data Availability}

The data used to support the findings of this study are available from the corresponding authors upon request. The sequence data generated in this study were submitted to the GenBank Sequence Read Archive accession number PRJNA540021.

\section{Disclosure}

The funders had no role in study design, data collection and analysis, decision to publish, or preparation of the manuscript.

\section{Conflicts of Interest}

The authors declare no conflicts of interest.

\section{Authors' Contributions}

YZ, LL, and YL designed and conceived the experiments. YG, $\mathrm{LL}$, and $\mathrm{ZH}$ performed the experiments. YG, XF, and JZ conducted the statistical analysis. YG, QM, and JF analyzed the data, prepared figures, and wrote the paper. $\mathrm{YZ}, \mathrm{CH}$, and YL reviewed the paper. All authors read and approved the final manuscript. Yuecheng Guo, Chunlan Huang, and Liyan Liu contributed equally to this work.

\section{Acknowledgments}

We would like to thank Ling Tian, Baokun He, and Yuhui Jiang for their help and technical assistance. This study was supported by funds from the Medical-Engineering Cross Project of Shanghai Jiao Tong University (YG2015MS29, http://kejichu.sjtu.edu.cn/), the Clinical Research Cultivating Program of Shanghai Hospital Development Center (SHDC12017X09), the Shanghai General Hospital Clinical Research Innovation Team Project (CTCCR-2016B03), the National Natural Science Foundation of China (No. 81970555), and the Foundation of Shanghai Jiao Tong University School of Medicine for the Research-oriented Doctors (No. 20181813).

\section{Supplementary Materials}

Supplementary Table 1: pancreatic lesion index (scoring criteria). Supplementary Table 2: ileal lesion index (scoring criteria). Supplementary Figure 1: dithizone depletes Paneth cells in rats. Supplementary Figure 2: effect of high-fat diet on ANP-associated injuries caused by retrograde sodium taurocholate infusion. Supplementary Figure 3: effects of dithizone in rats on standard diet followed by sham biliopancreatic infusion. Supplementary Figure 4: effects of dithizone in rats on high-fat diet followed by sham biliopancreatic infusion. Supplementary Figure 5: mechanism of Paneth cells in pancreatic and intestinal injuries. Supplementary Figure 6: 
images of the control reaction (tissue sections where the primary antibody was omitted during the immunohistochemistry reaction). (Supplementary Materials)

\section{References}

[1] J. Scherer, V. P. Singh, C. S. Pitchumoni, and D. Yadav, "Issues in hypertriglyceridemic pancreatitis: an update," Journal of Clinical Gastroenterology, vol. 48, no. 3, pp. 195-203, 2014.

[2] P. Valdivielso, A. Ramírez-Bueno, and N. Ewald, "Current knowledge of hypertriglyceridemic pancreatitis," European Journal of Internal Medicine, vol. 25, no. 8, pp. 689-694, 2014.

[3] C. Tan, Z. Ling, Y. Huang et al., "Dysbiosis of intestinal microbiota associated with inflammation involved in the progression of acute pancreatitis," Pancreas, vol. 44, no. 6, pp. 868875, 2015.

[4] J. Chen, C. Huang, J. Wang et al., "Dysbiosis of intestinal microbiota and decrease in Paneth cell antimicrobial peptide level during acute necrotizing pancreatitis in rats," PLoS One, vol. 12, no. 4, article e0176583, 2017.

[5] C. Huang, J. Chen, J. Wang et al., "Dysbiosis of intestinal microbiota and decreased antimicrobial peptide level in Paneth cells during hypertriglyceridemia-related acute necrotizing pancreatitis in rats," Frontiers in Microbiology, vol. 8, p. 776, 2017.

[6] Y. Kurashima, Y. Goto, and H. Kiyono, "Mucosal innate immune cells regulate both gut homeostasis and intestinal inflammation," vol. 43, pp. 3108-3115, 2013.

[7] T. Ayabe, D. P. Satchell, C. L. Wilson, W. C. Parks, M. E. Selsted, and A. J. Ouellette, "Secretion of microbicidal $\alpha$-defensins by intestinal Paneth cells in response to bacteria," Nature Immunology, vol. 1, no. 2, pp. 113-118, 2000.

[8] S. Bel, M. Pendse, Y. Wang et al., "Paneth cells secrete lysozyme via secretory autophagy during bacterial infection of the intestine," Science, vol. 357, no. 6355, pp. 1047-1052, 2017.

[9] J. Wehkamp, N. H. Salzman, E. Porter et al., "Reduced Paneth cell $\alpha$-defensins in ileal Crohn's disease," Proceedings of the National Academy of Sciences of the United States of America, vol. 102, no. 50, pp. 18129-18134, 2005.

[10] L. Schwingshackl and G. Hoffmann, "Comparison of effects of long-term low-fat vs high-fat diets on blood lipid levels in overweight or obese patients: a systematic review and metaanalysis," Journal of the Academy of Nutrition and Dietetics, vol. 113, no. 12, pp. 1640-1661, 2013.

[11] X. Guo, J. Li, R. Tang et al., "High fat diet alters gut microbiota and the expression of Paneth cell-antimicrobial peptides preceding changes of circulating inflammatory cytokines," Mediators of Inflammation, vol. 2017, Article ID 9474896, 9 pages, 2017.

[12] R. B. Canani, M. Di Costanzo, L. Leone, M. Pedata, R. Meli, and A. Calignano, "Potential beneficial effects of butyrate in intestinal and extraintestinal diseases," World Journal of Gastroenterology, vol. 17, no. 12, pp. 15191528, 2011.

[13] L. Zheng, C. J. Kelly, K. D. Battista et al., "Microbial-derived butyrate promotes epithelial barrier function through IL-10 receptor-dependent repression of claudin-2," The Journal of Immunology, vol. 199, no. 8, pp. 2976-2984, 2017.
[14] V. A. Matheus, L. C. S. Monteiro, R. B. Oliveira, D. A. Maschio, and C. B. Collares-Buzato, "Butyrate reduces high-fat dietinduced metabolic alterations, hepatic steatosis and pancreatic beta cell and intestinal barrier dysfunctions in prediabetic mice," Experimental Biology and Medicine, vol. 242, no. 12, pp. 1214-1226, 2017.

[15] M. Sawada, K. Takahashi, S. Sawada, and O. Midorikawa, "Selective killing of Paneth cells by intravenous administration of dithizone in rats," International Journal of Experimental Pathology, vol. 72, no. 4, pp. 407-421, 1991.

[16] J. Zheng, J. H. Wu, J. Chen et al., “Therapeutic effects of quercetin on early inflammation in hypertriglyceridemia-related acute pancreatitis and its mechanism," Pancreatology, vol. 16, no. 2, pp. 200-210, 2016.

[17] J. Schmidt, D. W. Rattner, K. Lewandrowski et al., "A better model of acute pancreatitis for evaluating therapy," Annals of Surgery, vol. 215, no. 1, pp. 44-56, 1992.

[18] C. J. Chiu, H. J. Scott, and F. N. Gurd, "Intestinal mucosal lesion in low-flow states. II. The protective effect of intraluminal glucose as energy substrate," Archives of Surgery, vol. 101, no. 4, p. 484, 1970.

[19] J. Zheng, X.-H. Xiao, Q. Zhang et al., "Correlation of placental microbiota with fetal macrosomia and clinical characteristics in mothers and newborns," Oncotarget, vol. 8, no. 47, pp. 82314-82325, 2017.

[20] L. Zhou, S. M. Limbu, M. Shen et al., "Environmental concentrations of antibiotics impair zebrafish gut health," Environmental Pollution, vol. 235, pp. 245-254, 2018.

[21] R. C. Edgar, B. J. Haas, J. C. Clemente, C. Quince, and R. Knight, "UCHIME improves sensitivity and speed of chimera detection," Bioinformatics, vol. 27, no. 16, pp. 21942200, 2011.

[22] C. Quast, E. Pruesse, P. Yilmaz et al., "The SILVA ribosomal RNA gene database project: improved data processing and web-based tools," Nucleic Acids Research, vol. 41, no. D1, pp. D590-D596, 2012.

[23] F. O. Glöckner, P. Yilmaz, C. Quast et al., "25 years of serving the community with ribosomal RNA gene reference databases and tools," Journal of Biotechnology, vol. 261, pp. 169-176, 2017.

[24] J. Zhang, X. Wang, D. Huo et al., "Metagenomic approach reveals microbial diversity and predictive microbial metabolic pathways in Yucha, a traditional Li fermented food," Scientific Reports, vol. 6, no. 1, article 32524, 2016.

[25] H.-N. Liu, T.-T. Liu, H. Wu et al., "Serum microRNA signatures and metabolomics have high diagnostic value in colorectal cancer using two novel methods," Cancer Science, vol. 109, no. 4, pp. 1185-1194, 2018.

[26] Y. Zeng, X. Wang, W. Zhang, K. Wu, and J. Ma, "Hypertriglyceridemia aggravates ER stress and pathogenesis of acute pancreatitis," Hepato-Gastroenterology, vol. 59, no. 119, pp. 2318-2326, 2012.

[27] Y. Murakami, S. Tanabe, and T. Suzuki, "High-fat dietinduced intestinal hyperpermeability is associated with increased bile acids in the large intestine of mice," Journal of Food Science, vol. 81, no. 1, pp. H216-H222, 2016.

[28] L. K. Stenman, R. Holma, and R. Korpela, "High-fat-induced intestinal permeability dysfunction associated with altered fecal bile acids," World Journal of Gastroenterology, vol. 18, no. 9, pp. 923-929, 2012.

[29] J. J. Song, W. J. Tian, L.-Y. Kwok et al., "Effects of microencapsulated Lactobacillus plantarum LIP-1 on the gut microbiota of 
hyperlipidaemic rats," British Journal of Nutrition, vol. 118, no. 7, pp. 481-492, 2017.

[30] A. Soggiu, C. Piras, S. L. Mortera et al., "Unravelling the effect of clostridia spores and lysozyme on microbiota dynamics in Grana Padano cheese: a metaproteomics approach," Journal of Proteomics, vol. 147, pp. 21-27, 2016.

[31] K. Cadwell, K. K. Patel, N. S. Maloney et al., "Virus-plus-susceptibility gene interaction determines Crohn's disease gene Atg16L1 phenotypes in intestine," Cell, vol. 141, no. 7, pp. 1135-1145, 2010.

[32] S. Fritz, T. Hackert, W. Hartwig et al., "Bacterial translocation and infected pancreatic necrosis in acute necrotizing pancreatitis derives from small bowel rather than from colon," The American Journal of Surgery, vol. 200, no. 1, pp. 111-117, 2010.

[33] W. S. Deng, J. Zhang, H. Ju et al., “Arpin contributes to bacterial translocation and development of severe acute pancreatitis," World Journal of Gastroenterology, vol. 21, no. 14, pp. 4293-4301, 2015.

[34] G. Capurso, G. Zerboni, M. Signoretti et al., "Role of the gut barrier in acute pancreatitis," Journal of Clinical Gastroenterology, vol. 46, pp. S46-S51, 2012.

[35] J. R. White, H. Gong, B. Pope, P. Schlievert, and S. J. McElroy, "Paneth-cell-disruption-induced necrotizing enterocolitis in mice requires live bacteria and occurs independently of TLR4 signaling," Disease Models \& Mechanisms, vol. 10, no. 6, pp. 727-736, 2017.

[36] A. Hanash, J. A. Dudakov, G. Hua et al., "Interleukin-22 protects intestinal stem cells from immune-mediated tissue damage and regulates sensitivity to graft versus host disease," Immunity, vol. 37, no. 2, pp. 339-350, 2012.

[37] I. I. Ivanov and D. R. Littman, "Modulation of immune homeostasis by commensal bacteria," Current Opinion in Microbiology, vol. 14, no. 1, pp. 106-114, 2011.

[38] Z. Zakostelska, M. Kverka, K. Klimesova et al., "Lysate of probiotic Lactobacillus casei DN-114 001 ameliorates colitis by strengthening the gut barrier function and changing the gut microenvironment," PLoS One, vol. 6, no. 11, article e27961, 2011.

[39] S. R. Llewellyn, G. J. Britton, E. J. Contijoch et al., "Interactions between diet and the intestinal microbiota alter intestinal permeability and colitis severity in mice," Gastroenterology, vol. 154, no. 4, pp. 1037-1046.e2, 2018.

[40] R. P. Dickson, B. H. Singer, M. W. Newstead et al., "Enrichment of the lung microbiome with gut bacteria in sepsis and the acute respiratory distress syndrome," Nature Microbiology, vol. 1, no. 10, article 16113, 2016.

[41] P. Xu, J. Wang, F. Hong et al., "Melatonin prevents obesity through modulation of gut microbiota in mice," Journal of Pineal Research, vol. 62, no. 4, article e12399, 2017.

[42] X. Wei, J. Tao, S. Xiao et al., "Xiexin Tang improves the symptom of type 2 diabetic rats by modulation of the gut microbiota," Scientific Reports, vol. 8, article 3685, 2018.

[43] U. D. Wankhade, Y. Zhong, P. Kang et al., "Enhanced offspring predisposition to steatohepatitis with maternal high-fat diet is associated with epigenetic and microbiome alterations," PLoS One, vol. 12, no. 4, article e0175675, 2017.
[44] M. H. Kim, S. G. Kang, J. H. Park, M. Yanagisawa, and C. H. Kim, "Short-chain fatty acids activate GPR41 and GPR43 on intestinal epithelial cells to promote inflammatory responses in mice," Gastroenterology, vol. 145, no. 2, pp. 396-406.e10, 2013.

[45] Y. Feng, Y. Wang, P. Wang, Y. Huang, and F. Wang, "Shortchain fatty acids manifest stimulative and protective effects on intestinal barrier function through the inhibition of NLRP3 inflammasome and autophagy," Cellular Physiology and Biochemistry, vol. 49, no. 1, pp. 190-205, 2018. 


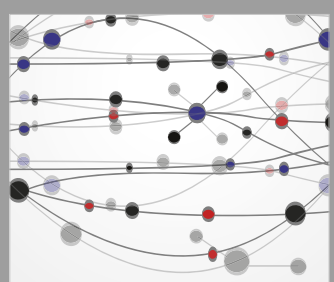

The Scientific World Journal
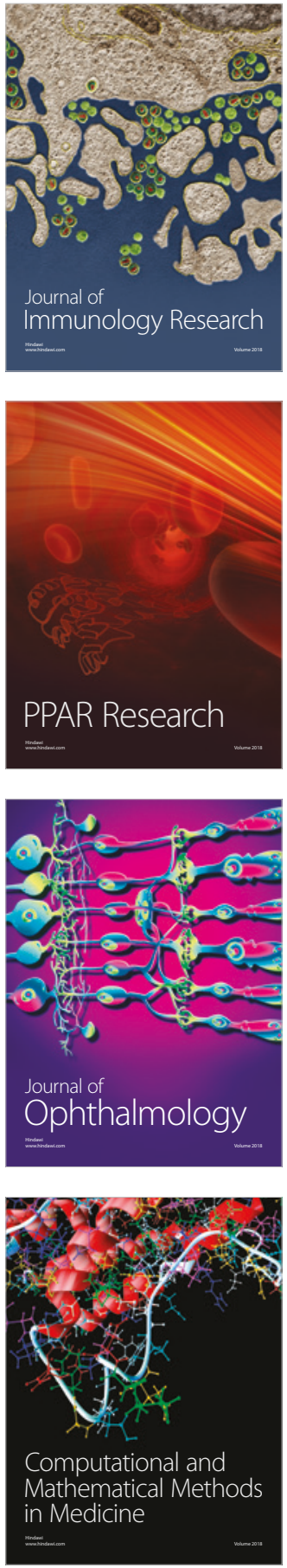

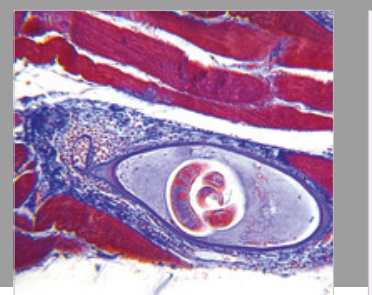

Gastroenterology Research and Practice

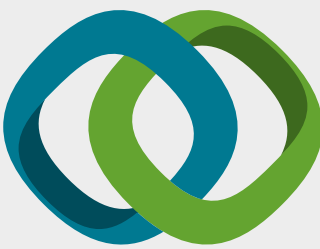

\section{Hindawi}

Submit your manuscripts at

www.hindawi.com
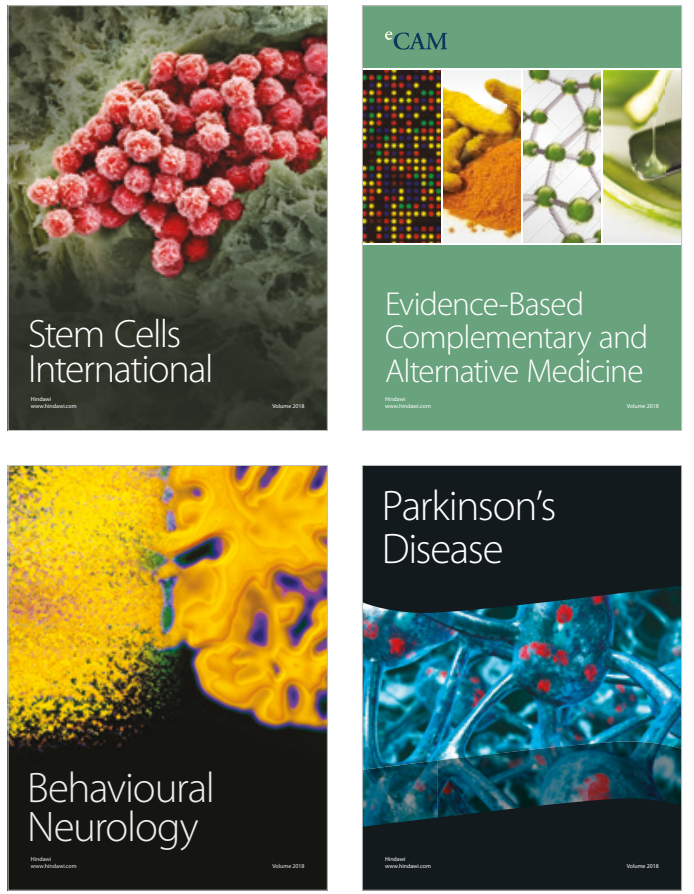

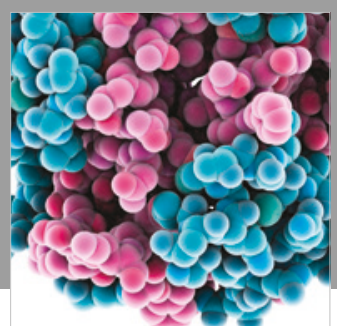

ournal of

Diabetes Research

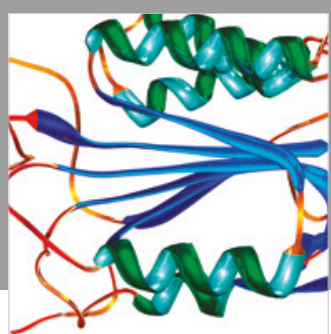

Disease Markers
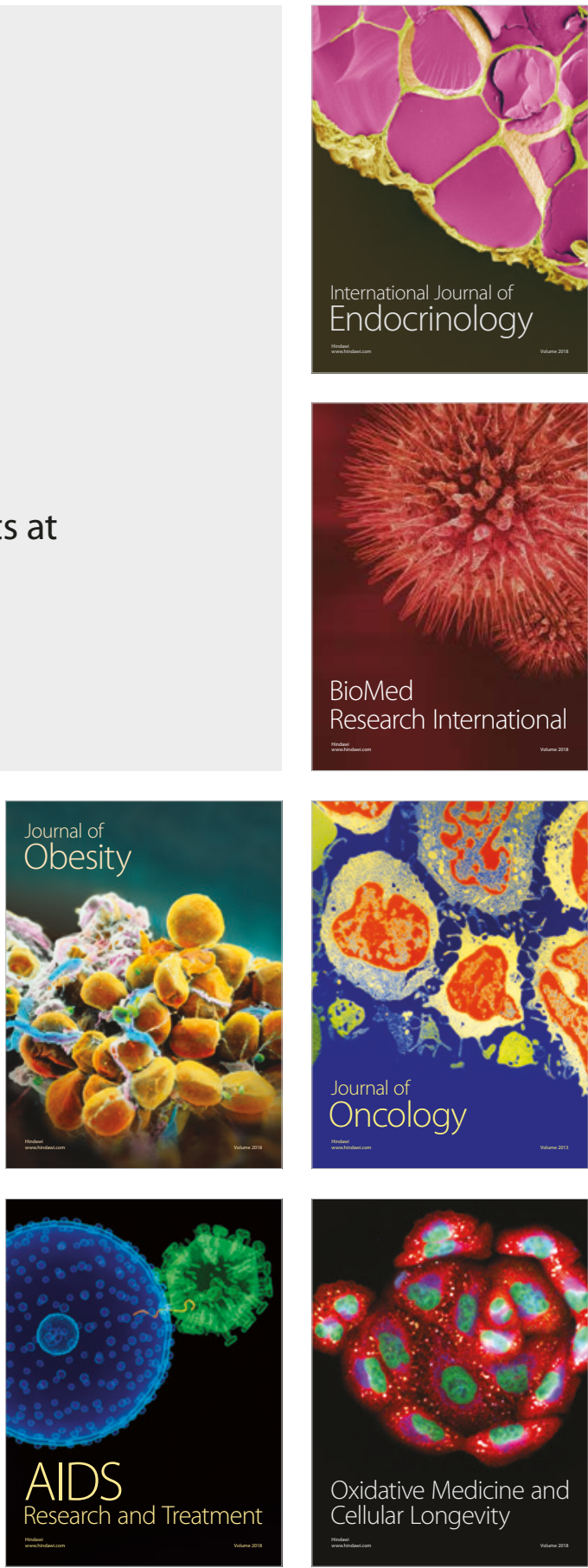